\title{
An Effective Model for Glueballs and Dual Superconductivity at Finite Temperature
}

\author{
Adamu Issifu $\left.{ }^{1}\right)^{1}$ and Francisco A. Brito ${ }^{1,2}$ \\ ${ }^{1}$ Departamento de Física, Universidade Federal da Paraíba, Caixa Postal 5008, 58051-970 João Pessoa, Paraíba, Brazil \\ ${ }^{2}$ Departamento de Física, Universidade Federal de Campina Grande, Caixa Postal 10071, 58429-900 Campina Grande, \\ Paraíba, Brazil
}

Correspondence should be addressed to Francisco A. Brito; fabrito@df.ufcg.edu.br

Received 21 May 2021; Accepted 17 July 2021; Published 10 August 2021

Academic Editor: Edward Sarkisyan Grinbaum

Copyright (c) 2021 Adamu Issifu and Francisco A. Brito. This is an open access article distributed under the Creative Commons Attribution License, which permits unrestricted use, distribution, and reproduction in any medium, provided the original work is properly cited. The publication of this article was funded by SCOAP ${ }^{3}$.

The glueballs lead to gluon and QCD monopole condensations as by-products of color confinement. A color dielectric function $G(|\phi|)$ coupled with a Abelian gauge field is properly defined to mediate the glueball interactions at confining regime after spontaneous symmetry breaking (SSB) of the gauge symmetry. The particles are expected to form through the quark-gluon plasma (QGP) hadronization phase where the free quarks and gluons start clamping together to form hadrons. The QCD-like vacuum $\left\langle\eta^{2} m_{\eta}^{2} F^{\mu \nu} F_{\mu \nu}\right\rangle$, confining potential $V_{c}(r)$, string tension $\sigma$, penetration depth $\lambda$, superconducting and normal monopole densities $\left(n_{s} n_{n}\right)$, and the effective masses $\left(m_{\eta}^{2}\right.$ and $\left.m_{A}^{2}\right)$ will be investigated at finite temperature $T$. We also calculate the strong "running" coupling $\alpha_{s}$ and subsequently the QCD $\beta$-function. The dual superconducting nature of the QCD vacuum will be investigated based on monopole condensation.

\section{Introduction}

The Large Hadron Collider (LHC) is currently attracting the attention of particle physicists to investigate the dynamics of the standard model (SM) at the TeV scale and to probe for a possible new physics. One of the major expectations at the LHC is the observation of jet substructures. Generally, jets are collimated bundles of hadrons constituted by quarks and gluons at short distances or high energies [1-3]. Jets played a significant role in the discovery of gluons $(g)$ [4-7] and top quark $(t)[8,9]$. These observations are featured prominently in classifying Quantum Chromodynamics (QCD) as a theory for strong interaction within the standard model. The most abundant secondary particles produced in heavy ion collisions are pions which emerge from hot and dense hadron gas states. These pions can be studied from the projected quark-gluon plasma (QGP) and the deconfinement phase through emitted photons and dileptons produced at this phase. Since the photon and the dilepton do not interact with the hadron matter through strong force, they decay quickly [10-13] making it easy to study the pions. Due to the large production of jets and pions at the LHC and Relativistic Heavy Ion Collider (RHIC), they are suitable candidates for the search for new particles and the testing of the SM properties. Quarks and gluons behave as quasi-free particles at higher energies (short distances) due to asymptotically free nature of the QCD theory. When the energy among these color particles is reduced to about $1 \mathrm{GeV}$ and below or at a separation of order $1 \mathrm{fm}$ or higher, color confinement sets in, such that the quarks and the gluons coexist as hadrons. At lower enough energies, hadronization is highly favoured leading to the formation of light pions. Hadron jet was first produced by annihilation of electron-positron to produce a two-jet event, $e^{+} e^{-} \longrightarrow q \bar{q} \longrightarrow 2$ - jets. This two-jet structure [14] was first observed in 1975 at SPEAR (SLAC), and the spin 1/2 nature of the quarks was also established [15]. The jets are produced when $q \bar{q}$ fly apart; more $q \bar{q}$ pairs are produced which recombine with the existing pairs to form mesons and baryons as a by-product [16]. The first three-jet structure from the electron-positron annihilation process 
was observed by analysing 40 hadronic events at the centerof-mass energy $27.4 \mathrm{GeV}$ from SPEAR [16]. These jets were subsequently observed clearly and separately $[17,18]$. The discovery of the gluon through the three-jet process played a major role in the discovery of the Higgs particle [19-24] by ATLAS collaboration [25] and CMS collaboration [26] using the LHC at CERN. On the other hand, pion-pion annihilation serves as the principal source of dileptons $\left(e^{-} e^{+}\right.$and $\mu^{-} \mu^{+}$) produced from the hadron matter [27, 28]. Thus, the proper study of the main source of dilepton spectra observed experimentally proposes significant observables which help to understand the pion dynamics in a dense nuclear matter that exist at the beginning of the collision [29].

We consider a complex scalar field model coupled to Abelian gauge fields in two different ways. At relatively low energies, the particles undergo transformation into glueballs. The emission of gluon $g$ which mediates the strong interactions is a non-Abelian feature observed in the nonperturbative regime of QCD theory. However, in the model framework, the gluon emission is a result of the modification of the Abelian gauge field by the color dielectric function. In this approach, we approximate the non-Abelian gauge field responsible for color confinement with an Abelian gauge field coupled with a dimensionless color dielectric function $G(|\phi|)$. The color dielectric function is responsible for regulating the long-distance dynamics of the photon propagator, so it does not decouple at higher wavelengths where glueballs are expected to be in a confined state. The color dielectric function is defined in terms of the glueball field $\eta$ after SSB, to give meaning to color confinement and its associated properties. While $\phi$ initiates the annihilation and production processes, $\eta$ is the glueball field which brings about confinement and gluon condensation. The Abelian approach to QCD theory was first proposed by 't Hooft to justify magnetic monopole condensation in QCD vacuum leading to color superconductivity $[30,31]$ that also suggested "infrared Abelian dominance." Fast forward, it has also been shown that about $92 \%$ of the QCD string tension is Abelian [32], so an Abelian approximation will not be out of place. Further studies of Abelian dominance for color confinement in SU(2) and SU(3) lattice QCD can be found in Ref. [33-37]. An interested reader can also refer to Ref. [38] for more recent results on Abelian dominance in QCD theory. This approach has also been adopted in other confinement models-see [39$41]$ and references therein for more justifications.

The annihilation of $\phi^{*} \phi$ through “scalar QED” where the particles are relatively free can be used to address hadronization due to the presence of high-density deconfined mater. The spontaneous symmetry breaking of the $U(1)$ symmetry to an energy regime is suitable for describing color confinement with a glueball field $\eta$. Because the running coupling, $\alpha_{s}\left(Q^{2}\right)$, of the strong interaction decreases with momentum increases (short distances), $\phi^{*} \phi$ annihilation can be studied from perturbation theory $\left(\phi^{*} \phi \longrightarrow \phi^{*} \phi\right)$ similar to annihilation and creation of pions $\left(\pi^{-} \pi^{+} \longrightarrow \pi^{-} \pi^{+} \gamma\right)$ and Bhabha scattering $\left(e^{-} e^{+} \longrightarrow e^{-} e^{+}\right)[42]$. A low energy analyses of $\phi^{*}$ $\phi$ annihilation will be made to show color confinement, bound states of the gluons (glueball masses) [43-45], and the QCD vacuum $[46,47]$ responsible for the gluon condensate. We will shed light on monopole condensation in the QCD vacuum and its role in screening the QCD vacuum from external electric and magnetic field penetrations similar to superconductors. There is a long-standing belief that quark masses are obtained through the spontaneous symmetry breaking mechanism facilitated by the nonzero expectation value of the Higgs field. That notwithstanding, quark masses continue to be an input parameter in the standard model [48]. As a free parameter in the SM, the magnitude of the mass is determined phenomenologically and the result compared with sum rules, lattice simulation results, other theoretical techniques, and experimental data. The Dyson-Schwinger integral equation for the quark mass function has two known solutions, i.e., trivial and nontrivial solutions. The latter is obtained under low energy and nonperturbative conditions while the former leads to unphysical, massless quarks. Thus, the nontrivial solution leads to the creation of quark masses resulting in "dynamical chiral symmetry breaking," which is a consequence of confinement [49-54]. Monopoles can be said to be a product of grand unification theory (GUT). The mixing of the strong and the electroweak interactions due to the higher gauge symmetries in GUT breaks spontaneously at higher energies or extremely short distances. So, physical features of monopoles such as size and mass are studied through the energy of spontaneous symmetry breaking [55]. Monopoles play important role in color confinement. Monopole condensation produces a dual superconductor which squeezes the uniform electric field at the confinement phase into a thin flux tube picture. The flux tube is formed between quark and an antiquark to keep them confined. This scenario has been extensively investigated in lattice gauge theory [56-58] and Polyakov loop [59] to establish its confinement properties. The involvement of monopoles in chiral symmetry has recently been investigated as well $[60,61]$. The impact of monopoles in quark-gluon plasma (QGP) has also been studied in [62-64].

The paper is organised as follows: In Section 2, we introduce the Lagrangian density that will be the basics for this study. In Section 3, we present spontaneous symmetry breaking of the model presented in the previous sections; this section is divided into four subsections; in Section 3.1, we investigate confinement of glueballs; in Section 3.2, we study the effective masses; we dedicate Section 3.3 to gluon condensation, and in Section 4, we investigate the monopole condensation. We proceed to present the analysis in Section 5.1 and the final findings in Section 5.2. We have adopted the natural units $c=\hbar=k_{B}=1$, except otherwise stated.

\section{The Model}

We start with a Lagrangian density developed by exploring gauge-invariant properties (see [39]):

$$
\begin{aligned}
\mathscr{L}= & \eta^{\mu \nu} D_{\mu} \phi D_{\nu} \phi^{*}-\frac{1}{4} G(|\phi|) F_{\mu \nu} F^{\mu \nu}-\frac{1}{4} \tilde{F}_{\mu \nu} \tilde{F}^{\mu \nu}-V(|\phi|) \\
= & \eta^{\mu \nu}\left(\partial_{\mu} \phi+i q \tilde{A}_{\mu} \phi\right)\left(\partial_{\nu} \phi^{*}-i q \tilde{A}_{\nu} \phi^{*}\right) \\
& -\frac{1}{4} G(|\phi|) F_{\mu \nu} F^{\mu \nu}-\frac{1}{4} \tilde{F}_{\mu \nu} \tilde{F}^{\mu \nu}-V(|\phi|)
\end{aligned}
$$


where $D_{\mu}=\partial_{\mu}+i q \tilde{A}_{\mu}, \tilde{F}_{\mu \nu}=\partial_{\mu} \tilde{A}_{v}-\partial_{v} \tilde{A}_{\mu}$, and $F_{\mu \nu}=\partial_{\mu} A_{v}$ $-\partial_{v} A_{\mu}$ are the covariant derivative, strength of the dual gauge field, and the gauge field, respectively. This model can be used to study the annihilation and creation of identical particles $\phi^{*} \phi$ and its transformation into glueballs $\eta$ at low energies. The complex scalar fields $\phi(x)$ and its conjugate $\phi^{*}(x)$ describe particle and its antiparticle with the same mass but different charges, respectively. The electromagnetic interactions among the scalar fields with an exchange of single photon produced from the dual gauge field dynamics $\widetilde{F}_{\mu \nu} \widetilde{F}^{\mu \nu}$ are the suitable scenario to address a high energy limit. However, we shall focus on the strong interactions, where the scalar fields modify to glueball fields at relatively low energies, which will lead to color confinement and its associated properties mediated by the modified gauge field dynamics $G(|\phi|) F_{\mu \nu} F^{\mu \nu}$. The equations of motion are

$$
\begin{gathered}
D_{\mu} D^{\mu} \phi+\frac{1}{4} \frac{\partial G(|\phi|)}{\partial \phi^{*}} F_{\mu \nu} F^{\mu \nu}+\frac{\partial V(|\phi|)}{\partial \phi^{*}}=0, \\
\partial_{\mu} \widetilde{F}^{\mu \nu}=-i q\left[\phi^{*} \partial^{v} \phi-\phi \partial^{v} \phi^{*}\right]+2 q^{2} \phi^{*} \phi \tilde{A}^{v}=j_{\phi}^{\nu}, \\
\partial_{\mu}\left[G(|\phi|) F^{\mu v}\right]=0 .
\end{gathered}
$$

We will adopt a complex scalar field potential of the form

$$
V(\phi)=\frac{\rho}{4}\left[\alpha^{2}|\phi|^{2}-a^{2}\right]^{2},
$$

where

$$
\phi=\frac{\phi_{1}+i \phi_{2}}{\sqrt{2}} .
$$

We can isolate the electromagnetic interactions among the fields [65] for study using Equation (3). This enables us to visualize how the fields annihilate and create identical particles $[66,67]$ through single-photon exchange, $\phi\left(p_{1}\right) \phi^{*}\left(p_{2}\right)$ $\longrightarrow \gamma \longrightarrow \phi\left(p_{1}^{\prime}\right) \phi^{*}\left(p_{2}^{\prime}\right)$, in high energy regime [68].

\section{Confinement, Effective Masses, Gluon Condensation, and Dual Superconductivity}

To discuss the color confinement and its consequences, we need to follow spontaneous symmetry breaking of the $\tilde{U}(1)$ symmetry in Lagrangian Equation (1) to give mass to the resulting glueball fields and their associated products such as the monopole condensation and gluon condensation which form the basics for color confinement. We will proceed with the symmetry breaking from the transformations:

$$
\begin{gathered}
\phi(x) \longrightarrow \phi^{\prime}(x)=e^{i q \alpha(x)} \phi, \\
\tilde{A}_{\mu}(x) \longrightarrow \tilde{A}_{\mu}^{\prime}(x)=\tilde{A}_{\mu}(x)-\partial_{\mu} \alpha(x) .
\end{gathered}
$$

The vacuum expectation value of the potential Equation
(5),

$$
\langle|\phi|\rangle_{0}= \pm \frac{a}{\alpha}
$$

breaks the $\tilde{U}(1)$ symmetry spontaneously. Considering two real scalar fields $\eta(r)$ and $\zeta(r)$ representing small fluctuations about the vacuum, a shift in the vacuum can be expressed as

$$
\phi \longrightarrow \eta(r)+\phi_{0}
$$

where $\phi_{0} \equiv\langle|\phi|\rangle_{0}$. Hence, the potential takes the form

$$
V(\eta)=\left.V(\phi)\right|_{\langle|\phi|\rangle_{0}}+\left.V^{\prime}(\phi)\right|_{\langle\mid \phi\rangle_{0}} \eta+\left.\frac{1}{2} V^{\prime \prime}(\phi)\right|_{\left\langle\langle\phi \mid\rangle_{0}\right.} \eta^{2}=\rho \alpha^{2} a^{2} \eta^{2} .
$$

Consequently, we can suitably parametrize the scalar field such that,

$$
\phi=e^{i \zeta / \phi_{0}} \frac{\left(\phi_{0}+\eta\right)}{\sqrt{2}} \approx \frac{\left(\phi_{0}+\eta(r)+i \zeta(r)\right)}{\sqrt{2}} .
$$

The Lagrangian in Equation (1) becomes

$$
\begin{aligned}
\mathscr{L}= & \frac{1}{2}\left[\partial_{\mu} \eta \partial^{\mu} \eta-2 \rho \alpha^{2} a^{2} \eta^{2}\right]+\frac{1}{2}\left[\partial_{\mu} \zeta \partial^{\mu} \zeta-2 q \phi_{0} \tilde{A}_{\mu} \partial^{\mu} \zeta+q^{2}\left|\phi_{0}\right|^{2} \tilde{A}_{\mu} \tilde{A}^{\mu}\right] \\
& -\frac{1}{4} G(\eta) F_{\mu v} F^{\mu v}-\frac{1}{4} \tilde{F}_{\mu v} \tilde{F}^{\mu v}+\cdots,
\end{aligned}
$$

where $\eta$ is associated with the glueball fields and $\zeta$ is also associated with the massless Goldstone bosons which will be swallowed by the massive gauge fields through the gauge transformation [39]:

$$
\tilde{A}_{\mu} \longrightarrow \tilde{A}_{\mu}^{\prime}=\tilde{A}_{\mu}-\frac{1}{q \phi_{0}} \partial_{\mu} \zeta .
$$

Pions are strongly interacting elementary particles with an integer spin. Therefore, they are bosons which are not governed by Pauli's exclusion principle and can exist as relativistic or nonrelativistic particles. As a result, they can be represented by spin- 0 scalar fields. In the nonrelativistic regime, they exist as Goldstone bosons $\zeta$, signifying the breakdown of chiral symmetry [69]. Pions are generally produced through matter annihilations such as $p \bar{p}, N \bar{N}$, and $e e^{+}$, which undergo transition from baryon structures to mesons. This is an interesting phenomenon in low energy hadron physics [70,71]. Pions have a flavour structure and other quantum numbers that permit us to classify them as bound states of quark and an antiquark. However, the valence quarks which characterize the flavour structure are surrounded by gluons and quark-antiquark pairs. Physically, glue-rich components mix with pions $(q \bar{q})$ forming an enriched spectrum of isospin-zero states and $q \bar{q} g$ hybrid states [72]. The glueball spectrum and their corresponding quantum numbers are known in lattice gauge theory 
predictions $[43,73]$. Additionally, the existence of glueballs has not been decisive because of the fear of possible mixing with quark degrees of freedom [74]. In the model framework, the glueballs coexist with the Goldstone bosons which are subsequently swallowed by the massive gauge fields leaving out the glueballs $(g g)$ for analysis. The glueball decay occurs when the separation distance between the valence gluons exceeds some thresholds ( $>1 \mathrm{fm}$ ) leading to hadronization.

Consequently, the Lagrangian can be simplified as

$$
\mathscr{L}=\frac{1}{2} \partial_{\mu} \eta \partial^{\mu} \eta-V(\eta)-\frac{1}{4} G(\eta) F_{\mu \nu} F^{\mu \nu}-\frac{1}{4} \tilde{F}_{\mu \nu} \tilde{F}^{\mu \nu}+\frac{q^{2}\left|\phi_{0}\right|^{2}}{2} \tilde{A}_{\mu} \tilde{A}^{\mu},
$$

where $V(\eta)=\rho \alpha^{2} a^{2} \eta^{2}$. The equations of motion for this Lagrangian are

$$
\begin{gathered}
\partial_{\mu} \partial^{\mu} \eta+\frac{\partial G(\eta)}{\partial \eta} F_{\mu \nu} F^{\mu \nu}+\frac{\partial V(\eta)}{\partial \eta}=0 \\
\partial_{\mu} \tilde{F}^{\mu \nu}=q^{2}\left|\phi_{0}\right|^{2} \tilde{A}^{v}=j_{\phi_{0}}^{v}, \\
\partial_{\mu}\left[G(|\eta|) F^{\mu \nu}\right]=0 .
\end{gathered}
$$

The Feynman propagator for the glueball field $\eta$ in Equation (12) reads

$$
D_{\eta}(p)=\frac{i}{p^{2}-m_{\eta}^{2}+i \varepsilon}
$$

where $m_{\eta}^{2}=2 \rho \alpha^{2} a^{2}$.

3.1. Confinement. Here, we are interested in calculating static confining potential, such that only chromoelectric flux that results in confining electric field is present, while chromomagnetic flux influence is completely eliminated. Thus, in spherical coordinates, Equations (15) and (17) become, respectively,

$$
\begin{aligned}
& \frac{1}{r^{2}} \frac{d}{d r}\left(r^{2} \frac{d \eta}{d r}\right)=\frac{1}{2} \frac{\partial G(\eta)}{\partial \eta} E^{2}-\frac{\partial V(\eta)}{\partial \eta}, \\
& \frac{1}{r^{2}} \frac{\partial}{\partial r}\left[r^{2} G(\eta) E\right]=0 \longrightarrow E=\frac{\Lambda}{r^{2} G(\eta)},
\end{aligned}
$$

where $\Lambda=q / 4 \pi \varepsilon_{0}$ is the integration constant. We are also now setting $G(\eta)=\xi^{2} V(\eta)$ (similar process was adopted in Ref. [39-41]), where $\xi^{2}$ is a dimensionful constant associated with the Regge slope $\left(2 \pi \alpha^{\prime}\right)^{2}$ that absorbs the dimensionality of $V(\eta)$, so $G(\eta)$ remains dimensionless and Equation (19) reads

$\frac{1}{r^{2}} \frac{d}{d r}\left(r^{2} \frac{d \eta}{d r}\right)=-\frac{\partial}{\partial \eta}\left[\frac{\Lambda^{2}}{2} \frac{1}{\xi^{2} V(\eta)} \frac{1}{r^{4}}+V\right] \longrightarrow \eta^{\prime \prime}+\frac{2}{r} \eta^{\prime}+m_{\eta}^{2} \eta=0$.

In the last step, we have assumed that the particles are far away from the charge source $q$, and in such a limit, terms of the order $O\left(1 / r^{4}\right)$ can be disregarded. This equation has a solution

$$
\eta(r)=\frac{a \sin \left(m_{\eta} r\right)}{\alpha m_{\eta} r}
$$
(20),

Substituting this result into the electric field Equation

$$
E=\frac{\Lambda}{r^{2} G}=\frac{2 \Lambda \alpha^{2}}{a^{2} \xi^{2} \sin ^{2}\left(m_{\eta} r\right)}
$$

and using the well-known expression for determining the electrodynamic potential,

$$
V(r)=\int E d r
$$

to determine the confining potential $V_{c}(r)$, we get

$$
\begin{aligned}
V_{c}(r) & =-\frac{2 \Lambda \alpha^{2} \cot \left(m_{\eta} r\right)}{a^{2} m_{\eta} \xi^{2}}+c \simeq-\frac{2 \Lambda \alpha^{2}}{a^{2} m_{\eta} \xi^{2}}\left[\frac{1}{m_{\eta} r}-\frac{m_{\eta} r}{3}-O\left(r^{3}\right)\right]+c \\
& \simeq \frac{2 \alpha^{2}}{a^{2} \xi^{2} m_{\eta}^{2}}\left[-\frac{1}{r}+\frac{m_{\eta}^{2} r}{3}\right] ;
\end{aligned}
$$

so, setting

$$
a^{4} \xi^{2} \rho=1 \longrightarrow \xi^{2}=\frac{1}{a^{4} \rho}
$$

leads to

$$
V_{c}(r)=-\frac{1}{r}+\frac{m_{\eta}^{2} r}{3}
$$

with string tension

$$
\sigma=\frac{m_{\eta}^{2}}{3}=\frac{2 \rho \alpha^{2} a^{2}}{3}
$$

In the last step of Equation (25), we substituted $\Lambda=q / 4$ $\pi \varepsilon_{0}=1$ and $c=0$ for simplicity.

3.2. Effective Masses. Glueballs are simply bound states of gluons, and they are "white" or colorless in nature. They are flavour blind and are capable of decaying. Scalar glueball with quantum number $J^{P C}=0^{++}$is observed by lattice QCD simulations as the lightest with a mass of about $1.7 \mathrm{GeV}$ [43-45].

The dispersion relation for the glueball and the gluon excitations including thermal fluctuations can be expressed 
as

$$
\begin{aligned}
& E_{\eta}^{2}=k^{2}+m_{\eta}^{* 2}, \\
& E_{A}^{2}=k^{2}+m_{A}^{* 2} .
\end{aligned}
$$

We can calculate the effective thermal fluctuating glueball mass by taking the second derivative of the Lagrangian density Equation (14) with respect to $\eta$ :

$$
m_{\eta}^{* 2}=-\left\langle\frac{\partial^{2} \mathscr{L}}{\partial \eta^{2}}\right\rangle=2 \rho \alpha^{2} a^{2}+\frac{1}{2} \rho \alpha^{2} a^{2} \xi^{2}\left\langle F^{\mu \nu} F_{\mu \nu}\right\rangle .
$$

We redefine the glueball field $\eta$ to include the thermal fluctuations as $\eta=\bar{\eta}+\Delta$, with restriction, $\langle\Delta\rangle=0$. The angle brackets represent thermal average, and $\bar{\eta}$ is the mean field of the glueballs. This equation can be solved by determining explicitly the nature of $\left\langle F^{\mu v} F_{\mu \nu}\right\rangle$ and $\left\langle\Delta^{2}\right\rangle$ using field quantum distributions. By the standard approach, we can express

$$
\begin{gathered}
\left\langle F^{\mu \nu} F_{\mu \nu}\right\rangle=-\frac{v}{2 \pi^{2}} \int_{0}^{\infty} d k \frac{k^{4}}{E_{A}} n_{B}\left(E_{A}\right), \\
\left\langle\Delta^{2}\right\rangle=\frac{1}{2 \pi^{2}} \int_{0}^{\infty} d k \frac{k^{2}}{E_{\eta}} n_{B}\left(E_{\eta}\right) .
\end{gathered}
$$

Here, $n_{B}(x)=\left(e^{x \beta}-1\right)^{-1}$ represents the Bose-Einstein distribution function and $\beta=1 / T$, where $T$ is the temperature. We can analytically solve Equation (31) at a high energy regime, where $E \approx k c, k_{B} T \gg m_{A} c^{2}$ and $m_{A}$ is the gluon/screening mass. Therefore,

$$
\left\langle F^{\mu \nu} F_{\mu \nu}\right\rangle=-\frac{v}{2 \pi^{2}} \int_{0}^{\infty} d k \frac{k^{4}}{E_{A}} \frac{1}{e^{\beta E_{A}-1}}=-\frac{v T^{4}}{2 \pi^{2}} \int_{0}^{\infty} \frac{x^{3} d x}{e^{x}-1}=-\frac{4 T^{4}}{T_{c}^{4} \xi^{2}},
$$

where we have substituted $x=k / T$ and used the standard integral

$$
\int \frac{x^{3} d x}{e^{x}-1}=\frac{\pi^{4}}{15}
$$

In the last step, we have substituted

$$
T_{c}^{4} \xi^{2}=\frac{15}{2 \pi^{2} v}
$$

being $T_{c}$ the critical temperature. Following the same analysis,

$$
\left\langle\Delta^{2}\right\rangle=\frac{1}{2 \pi^{2}} \int_{0}^{\infty} d k \frac{k^{2}}{E_{\eta}} \frac{1}{e^{\beta E_{\eta}}-1} \simeq \frac{T^{2}}{2 \pi^{2}} \int_{0}^{\infty} \frac{x d x}{e^{x}-1} \simeq \frac{T^{2}}{12}
$$

where we have assumed $k_{B} T \gg m_{\eta} c^{2}$, with $m_{\eta}$ being the glue- ball mass, and considered the standard integral,

$$
\int \frac{x d x}{e^{x}-1}=\frac{\pi^{2}}{6}
$$

Thus, Equation (30) becomes

$$
m_{\eta}^{* 2}(T)=2 \rho \alpha^{2} a^{2}\left[1-\frac{T^{4}}{T_{c}^{4}}\right]=m_{\eta}^{2}(0)\left[1-\frac{T^{4}}{T_{c}^{4}}\right]
$$

When we take a thermal average of Equation (15), we get $\xi^{2} m_{\eta}^{2}\left\langle\eta F^{\mu \nu} F_{\mu \nu}\right\rangle=-m_{\eta}^{2}\langle\eta\rangle$; therefore, $\bar{\eta}=0$ and $\bar{\eta}=1$ are exact solutions of the glueball fields [75]. We further demonstrate in Figures 1 and 2 that $\bar{\eta}$ has solution $\bar{\eta}=0$ at $T=0$ and increases steady as $T$ increases to its maximum $\bar{\eta} \simeq 1$ at $T=$ $T_{c}$, corresponding to no glueball fields and the melting of the glueballs.

3.3. Gluon Condensation. Classical gluodynamics is described by the Lagrangian density:

$$
\mathscr{L}^{\prime}=-\frac{1}{4} F^{a \mu \nu} F_{\mu \nu}^{a}
$$

which is invariant under scale and conformal symmetries, $x$ $\longrightarrow \lambda x$, and also produces vanishing gluon condensate \langle $\left.F^{a \mu \nu} F_{\mu \nu}^{a}\right\rangle=0$. Meanwhile, the symmetries are broken when quantum correction $-\left|\varepsilon_{v}\right|$ is added to the Lagrangian, i.e.,

$$
\mathscr{L}^{\prime}=-\frac{1}{4} F^{a \mu \nu} F_{\mu \nu}^{a}+\left|\varepsilon_{v}\right|
$$

resulting in nonvanishing gluon condensate, $\left\langle F^{a \mu v} F_{\mu \nu}^{a}\right\rangle>0$. This correction is the consequence of the well-known scale anomaly observed in QCD energy-momentum $\left(\theta^{\mu \nu}\right)$ trace

$$
\theta_{\mu}^{\mu}=\frac{\beta(g)}{2 g} F^{a \mu \nu} F_{\mu \nu}^{a}
$$

where $\beta(g)$ is the "so-called" beta-function of the strong coupling $g$, with a leading term

$$
\beta(g)=-\frac{11 g^{3}}{(4 \pi)^{2}} .
$$

Accordingly, we have nonzero vacuum expectation, expressed as

$$
\left\langle\theta_{\mu}^{\mu}\right\rangle=-4\left|\varepsilon_{v}\right|
$$

The second term in Equation (14) acts similar to the quantum correction and explicitly breaks the scale and the conformal symmetries, so its energy-momentum tensor satisfies Equation (42) [40, 76-78]. We will compute the trace of the energy-momentum tensor of Equation (14) using the 


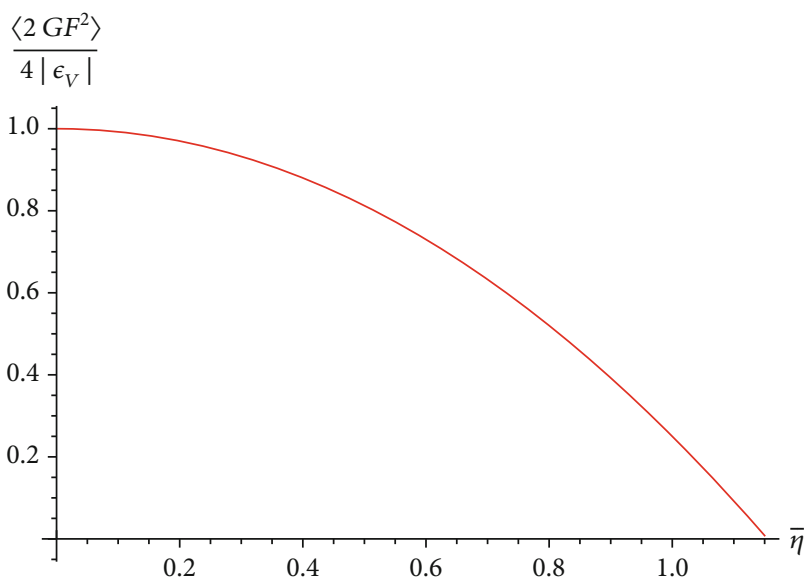

Figure 1: The gluon condensate with the mean glueball field $\bar{\eta}$. The condensate has its maximum value at $\bar{\eta}=0$ and vanishes at $\bar{\eta} \simeq 1$.

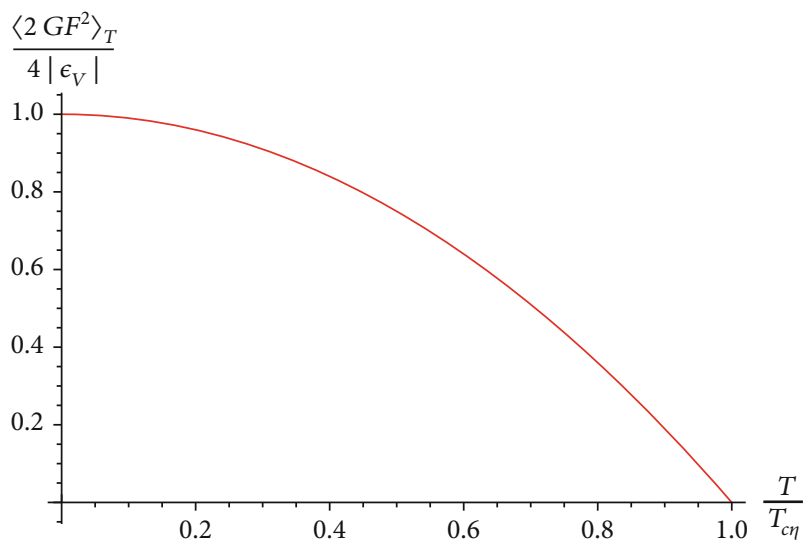

Figure 2: The gluon condensate with varying temperature $T$. At $T$ $=0, \quad\left\langle 2 G(\eta) F^{\mu \nu} F_{\mu \nu}\right\rangle_{T} / 4\left|\varepsilon_{v}\right|=1 \quad$ representing maximum condensate corresponding to $\bar{\eta}=0$. Also, at $T=T_{c}$, $\left\langle 2 G(\eta) F^{\mu \nu} F_{\mu \nu}\right\rangle_{T} / 4\left|\varepsilon_{v}\right|=0$ representing minimum condensate corresponding to $\bar{\eta} \simeq 1$ [75].

relation

$$
\theta_{\mu}^{\mu}=4 V(\eta)+\eta \square \eta
$$

Substituting the equation of motion Equation (15) into Equation (43) yields

$$
\theta_{\mu}^{\mu}=4 V(\eta)-\eta \frac{\partial G}{\partial \eta} F^{\mu \nu} F_{\mu \nu}-\eta \frac{\partial V}{\partial \eta}=4 \tilde{V}-\eta G^{\prime} F^{\mu \nu} F_{\mu \nu}
$$

where in the last step we have substituted $\tilde{V}=V(\eta)-\eta V^{\prime}($ $\eta$ )/4 with $G^{\prime}$ and $V^{\prime}$ representing the derivative of $G$ and $V$ with respect to $\eta$, respectively. In order to make the expression easy to be compared with Equation (42), we rescale $\tilde{V}$ as $\tilde{V}(\eta) \longrightarrow-\left|\varepsilon_{v}\right| \tilde{V}$, consequently,

$$
\left\langle\eta G^{\prime} F^{\mu v} F_{\mu \nu}\right\rangle=4\left|\varepsilon_{v}\right|\langle 1-\tilde{V}\rangle \text {. }
$$

We recover the classical expression for $\left\langle F^{\mu \nu} F_{\mu \nu}\right\rangle=0$ when we set $\left|\varepsilon_{v}\right| \longrightarrow 0$. Following the potential defined below Equation (14), we can express

$$
\tilde{V}=V-\frac{\eta V^{\prime}}{4}=\frac{\rho a^{2} \alpha^{2} \eta^{2}}{2}=\frac{m_{\eta}^{2} \eta^{2}}{4}
$$

thus, Equation (45) becomes

$$
\left\langle 2 G(\eta) F^{\mu \nu} F_{\mu \nu}\right\rangle=4\left|\varepsilon_{v}\right|\left\langle 1-\frac{m_{\eta}^{2} \eta^{2}}{4}\right\rangle .
$$

The QCD vacuum is seen as a very dense state of matter comprising gauge fields and quarks that interact in a haphazard manner. These characteristics are hard to be seen experimentally because quark and gluon fields cannot be observed directly; only the color neutral hadrons are observable. The appearance of the mass term in the condensate is also significant because it leads to chiral symmetry breakdown in the vacuum. Additionally, the gluon mass $m_{A}$ can be derived from the vacuum as a function of the glueball mass as

$$
m_{A}^{2}=\frac{m_{\eta}^{2}}{4}
$$

Generally, scalar glueball mass is related to the gluon mass as

$$
\frac{m\left(0^{++}\right)}{m_{A}}=\sqrt{6} \cong 2.45
$$

considering the leading order $[79,80]$ from Yang-Mills theory with an auxiliary field $\phi$, here, the scalar glueball mass $m\left(0^{++}\right)$represents fluctuations around $\phi$. The gluon mass is determined to be $m_{A}=600 \sim 700 \mathrm{MeV}$ as obtained from lattice simulations [81-83]. Heavier gluon masses have also been observed in the range of $\sim 1 \mathrm{GeV}$ by phenomenological analysis [84, 85], lattice simulation [86], and analytical investigations [87]. The glueball mass $m_{\eta}$ is responsible for all the confinement properties and the chiral symmetry breaking in the vacuum. Observing that the string tension is $\sigma \sim 1$ $\mathrm{GeV} / \mathrm{fm}$, we can identify $m_{\eta}^{2}=3 \mathrm{GeV}^{2}$, corresponding to a gluon mass $m_{A}=0.87 \mathrm{GeV}$, from the model framework.

In terms of temperature, we use Equation (35), and the gluon condensate becomes

$$
\begin{aligned}
\left\langle 2 G(\eta) F^{\mu \nu} F_{\mu \nu}\right\rangle & =4\left|\varepsilon_{v}\right|\left[1-\frac{m_{\eta}^{2}}{4}\left(\frac{\phi_{0}^{2} T^{2}}{\phi_{0}^{2} 12}\right)\right]=4\left|\varepsilon_{v}\right|\left[1-\frac{T^{2}}{T_{c \eta}^{2}}\right], \quad \text { where } T_{c \eta} \\
& =\left(\frac{48}{m_{\eta}^{2}}\right)^{1 / 2} .
\end{aligned}
$$

Corresponding to a temperature fluctuating gluon mass

$$
m_{A}^{* 2}=\frac{m_{\eta}^{2} T^{2}}{48 \phi_{0}^{2}}=\tilde{g}^{2} T^{2}
$$


where $\tilde{g}^{2}=m_{\eta}^{2} / 48 \phi_{0}^{2}$ is a dimensionless coupling constant. This expression looks like the Debye mass derived from the leading order of QCD coupling expansion [88-90]. It carries nonperturbative property of the theory. Comparing Equations (40) and (44), we identify

$$
\frac{\beta(g)}{2 g}=-\eta G^{\prime}(\eta) \longrightarrow \beta\left(\frac{1}{r^{2}}\right)=-2 g \eta G^{\prime}(\eta) .
$$

Additionally, we can determine the strong running coupling $\alpha_{s}$ from the renormalization group theory [91]

$$
\beta\left(Q^{2}\right)=Q^{2} \frac{d \alpha_{s}}{d Q^{2}}
$$

therefore,

$\beta(\eta) \simeq-\eta \frac{d(G)}{d \eta}=-\xi^{2} m_{\eta}^{2} \eta^{2}(r)=2 G(\eta)=\beta\left(\frac{1}{r^{2}}\right)$ with $g=1$.

Comparatively, the strong running coupling can be identified as $\alpha_{s}(\eta)=G(\eta)=\alpha_{s}\left(1 / r^{2}\right)$, with $Q \equiv 1 / r$, as the spacelike momentum associated with the four-vector momentum as $Q^{2} \equiv-q^{2}$. Thus, the color dielectric function is associated with the QCD $\beta$-function and the strong running coupling. We can write the Feynman propagator for the interaction by considering the left-hand side of Equation (45):

$$
\begin{aligned}
F^{\mu v} F_{\mu v} & =-2 A^{v}\left[\square g_{\mu \nu}-\partial_{\mu} \partial_{v}\right] A^{\mu}=-2 A^{v}\left[\partial^{2} g_{\mu v}-\left(1-\frac{1}{\alpha}\right) \partial_{\mu} \partial_{v}\right] A^{\mu} \\
& =-2 A_{v}(k)\left[k^{2} g^{\mu v}-\left(1-\frac{1}{\alpha}\right) k^{\mu} k^{v}\right] A_{\mu}(-k) \longrightarrow \eta G^{\prime}(\eta) F^{\mu v} F_{\mu v} \\
& =-2 A_{v}(k) \eta G^{\prime}(\eta)\left[k^{2} g^{\mu v}-\left(1-\frac{1}{\alpha}\right) k^{\mu} k^{v}\right] A_{\mu}(-k) \\
& =A_{v}(k) \beta\left(q^{2}\right)\left[k^{2} g^{\mu v}-\left(1-\frac{1}{\alpha}\right) k^{\mu} k^{v}\right] A_{\mu}(-k),
\end{aligned}
$$

where we have added the gauge fixing term $(2 / \alpha)\left(\partial_{\mu} A^{\mu}\right)^{2}$ and made the Fourier transform

$$
A_{\mu}(k)=\int \frac{d^{4} k}{(2 \pi)^{4}} e^{-i k x} A_{\mu}(x) .
$$

In the last step, the term in the square brackets is precisely the photon propagator normally associated with Abelian gauge fields, but the multiplicative factor $-2 \eta G^{\prime}(\eta)=\beta\left(1 / r^{2}\right) \equiv \beta\left(q^{2}\right)$ is defined such that the photon propagator does not decouple at higher wavelengths. This influences the photons to behave like gluons.

Also, one can retrieve all the major results obtained in [40], if we consider temperature fluctuations in the scalar field $\phi$. In that case, we move away from the known form of field theory, i.e., perturbation about the vacuum, $\langle\phi\rangle=0$, to account for the density of particles and their interactions about the vacuum and the thermal distributions in the scalar field. So, we can substitute $\phi \longrightarrow \phi+\phi_{T}$ into the potential in Equation (5), where $\phi_{T}$ plays a similar role as $\Delta$ introduced above. Thus,

$$
\left\langle\phi_{T}^{2}\right\rangle=\frac{1}{2 \pi^{2}} \int_{0}^{\infty} \frac{p^{2} d p}{E\left(e^{E \beta}-1\right)} \simeq \frac{T^{2}}{12} ;
$$

here, we have used the high-temperature approximation $k_{B}$ $T \gg m_{\phi} c^{2}, E \approx p c$ and the potential becomes

$$
\begin{aligned}
V(\phi, T) & =\frac{\rho}{4}\left[\alpha^{2}|\phi|^{2}+\alpha^{2}\left\langle\phi_{T}^{2}\right\rangle-a^{2}\right]^{2}=\frac{\rho}{4}\left[\alpha^{2}|\phi|^{2}-a^{2}\left(1-\frac{T^{2}}{T_{c \phi}^{2}}\right)\right]^{2} \\
& =\frac{\rho}{4}\left[\alpha^{2}|\phi|^{2}-\tilde{a}^{2}\right]^{2},
\end{aligned}
$$

where $\left\langle\phi_{T}\right\rangle=0$,

$$
\begin{gathered}
T_{c \phi}^{2}=\frac{12 a^{2}}{\alpha^{2}}, \\
\tilde{a}^{2}=a^{2}\left(1-\frac{T^{2}}{T_{c \phi}^{2}}\right) .
\end{gathered}
$$

The glueball mass can be calculated from the fluctuations in Equation (58) around the vacuum, such that

$$
m_{\phi}^{* 2}(T)=\left.\frac{\partial^{2} V}{\partial \phi^{2}}\right|_{\phi_{0}}=2 \rho \alpha^{2} \tilde{a}^{2}=2 \rho \alpha^{2} a^{2}\left[1-\frac{T^{2}}{T_{c \phi}^{2}}\right]=m_{\eta}^{2}(0)\left[1-\frac{T^{2}}{T_{c \phi}^{2}}\right],
$$

where $\phi_{0}=\tilde{a} / \alpha$ is the vacuum of the potential. We observe that $m_{\phi}^{* 2}(0)=m_{\eta}^{* 2}(0)$ at $T=0$, similar to Equation (37). The differences in the degree of the temperature in both equations arise because Equation (37) has temperature correction to the gauge field $\left\langle F^{\mu \nu} F_{\mu \nu}\right\rangle$, while in Equation (37), we have temperature correction to the scalar field $\left\langle\phi_{T}^{2}\right\rangle$. Following the same procedure as the one used in deriving Equation (47), we can express

$$
\begin{aligned}
\left\langle 2 G(\eta) F^{\mu \nu} F_{\mu \nu}\right\rangle & =-4|\varepsilon|\left\langle\frac{m_{\phi}^{* 2}(T) \eta^{2}}{4}-1\right\rangle \\
& =-4|\varepsilon|\left\langle\frac{m_{\phi}^{2}(0)}{4}\left[1-\frac{T^{2}}{T_{c \phi}^{2}}\right] \eta^{2}-1\right\rangle .
\end{aligned}
$$

Here, we have replaced $m_{\eta}^{* 2}(T)$ with $m_{\phi}^{* 2}(T)$ from Equation (47). The negative sign and $\eta^{2}$ differences between this result and Ref. [40] might be due to the differences in the methods adopted for calculating the energy-momentum trace tensor $\theta_{\mu}^{\mu}$. Also, if we consider the IR part of the potential in Equation (25) as studied in Ref. [40], the potential 
becomes

$$
V_{c}(r)=\sigma r=\frac{m_{\phi}^{2}}{3} r \longrightarrow V_{c}(T, r)=\frac{m_{\phi}^{* 2}(T)}{3} r=\frac{m_{\phi}^{2}(0)}{3}\left[1-\frac{T^{2}}{T_{c \phi}^{2}}\right] r
$$

with string tension

$$
\sigma(T)=\frac{m_{\phi}^{* 2}(T)}{3}
$$

which is similar to the result in Ref. [40]. Therefore, the approach adopted in this paper accounts for the temperature corrections to both the glueball field $\left\langle\Delta^{2}\right\rangle$ in Equation (35) and the gauge field $\left\langle F^{\mu \nu} F_{\mu \nu}\right\rangle$, while in Ref. [40], only the thermal correction to the scalar field $\left\langle\phi_{T}^{2}\right\rangle$ was considered.

\section{Dual Superconductivity}

In this section, we will discuss briefly color superconductivity and proceed to elaborate on dual superconductivity in detail. The fields $\phi^{*} \phi$ annihilate and create identical fields through a decay process, $\phi\left(p_{1}\right) \phi^{*}\left(p_{2}\right) \longrightarrow \phi\left(p_{1}^{\prime}\right) \phi^{*}\left(p_{2}^{\prime}\right)$. The dual gauge field $\tilde{F}^{\mu \nu} \tilde{F}_{\mu \nu}$ which mediates the interaction of the scalar fields $\phi$ during the annihilation process is also responsible for the monopole condensation. In the model framework, the scalar field can be seen as point-like, asymptotically free and degenerate at the high energy regime [92]. In the context of this work, the high energy regime is in reference to the phase at which the scalar fields are annihilating while low energy regime is after the symmetry breaking or the glueball regime. In effect, multiparticle states are formed in the high energy regime. In such a high particle density regime, the charged scalar fields form Bose-Einstein condensation [93, 94] similar to induced isospin imbalance systems leading to color superconductivity $[95,96]$. With this type of condensation, there is a higher occupation number at the ground state than the excited states. So, temperature increase goes into reducing the occupation numbers [97]. There are several works on nonzero isospin chemical potential $\left(\mu_{I}\right)$ and baryon chemical potential $\left(\mu_{B}\right)$ in pion condensation [98-100], and they are related to the two flavour quarks that constitute the pions as $\mu_{I}=\left(\mu_{u}-\mu_{d}\right) / 2$ and $\mu_{B}=\left(\mu_{u}+\mu_{d}\right) / 2$, respectively. It has also been established [101] analytically at the quark level that the critical isospin chemical potential [102, 103] for pion superfluidity is precisely the pion mass $\mu_{I}^{c}=m_{\pi}$ in vacuum. This behaviour and other related isospin chemical potentials in pion condensate have been investigated using the Nambu-Jona-Lasinio (NJL) model, ladder-QCD [98].

In the model framework, the color superconducting property can be studied from the propagator, $\left\langle\phi \phi^{*}\right\rangle$. The high density region where the particles are asymptotically free forms deconfined matter leading to Bose-Einstein condensation resulting in the color superconducting phase [96, $104,105]$. On the other hand, at the low energy region, the fields modify into glueballs with real mass, $m_{\eta}$, leading to chiral symmetry breaking and color confinement. Since quark- quark combinations do not form color singlet, Cooper pair condensate will break the local color symmetry giving rise to color superconductivity [106]. This phenomenon was first studied by Bardeen, Cooper, and Schrieffer (BCS) [107]. The BCS mechanism for pairing in general appears to be more robust in dense quark matter than superconducting metals due to the nature of interactions among quarks. The virtual fermions in the vacuum make it color diamagnetic preventing both the electric and magnetic fields from penetrating [2].

We turn attention to the dual superconductivity in color confinement whose property we can derive from Equation (16). This subject was first proposed by Nambu, ' $t$ Hooft, and Mandelstam [56]. While the color superconducting phase takes place at the deconfined matter regime, the dual superconductivity is observed at the confining phase [108]. In the model framework, dual superconductivity is observed at the high glueball condensation region, $\eta \longrightarrow 0$, i.e., a region of strong confinement. We will use the rest of this section to discuss dual superconductivity in detail within the model framework. In this phenomenon, the QCD vacuum is seen as a color magnetic monopole condensate resulting in onedimensional squeezing of the uniform electric flux that form on the surface of the quark and antiquark pairs by dual Meissner effect. This results in the formation of a color flux tube between quark and antiquark used in describing string pictures of hadrons [38]. In this regard, magnetic monopole condensation is crucial to color confinement and dual superconductivity. Monopoles also play a role in chiral symmetry breaking [109] and strongly coupled QGP. They are involved in the decay of plasma formed immediately after relativistic heavy ion collision [62-64]. Even though monopoles have not been seen or proven experimentally, there are many theoretical bases for which one can believe their existence. The theoretical evidence for its existence is as strong as any undiscovered theoretical particle. Polyakov [110] and 't Hooft [111] discovered that monopoles are the consequences of the general idea of unification of fundamental interactions at short distances (high energies). Dirac on the other hand demonstrated the presence of monopoles in QED. Monopoles in general play an insightful role in understanding color confinement. They also provide some useful explanations to the features of superstring theory and supersymmetric quantum theory where the use of duality is a common phenomenon. The discovery of these monopoles some day will be interesting and possibly revolutionary since almost all existing technologies are based on electricity and magnetism.

Monopole condensation can now be exploited from the condensation of particles in the region $r \longrightarrow r_{*}$, where $\left\langle\phi \phi^{*}\right.$ \rangle$\sim\left\langle|\phi|^{2}\right\rangle_{0}$. Hence, from Equation (16), we can express

$$
\partial_{\mu} \tilde{F}^{\mu \nu}=q^{2}\left|\phi_{0}\right|^{2} \tilde{A}^{\nu}=j_{m}^{\nu}
$$

The equations of motion for the static fields are

$$
\begin{gathered}
\nabla \cdot \overrightarrow{\tilde{B}}=\rho_{m}, \\
-\nabla \times \overrightarrow{\widetilde{E}}=\vec{j}_{m} ;
\end{gathered}
$$




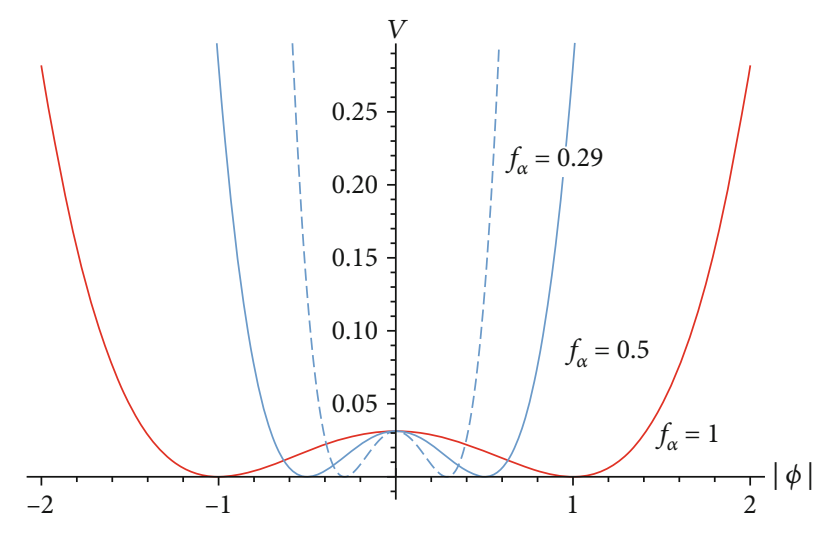

FIGURE 3: The scalar field potential for different decay constants $f_{\alpha}$. The potential has degenerate vacua.

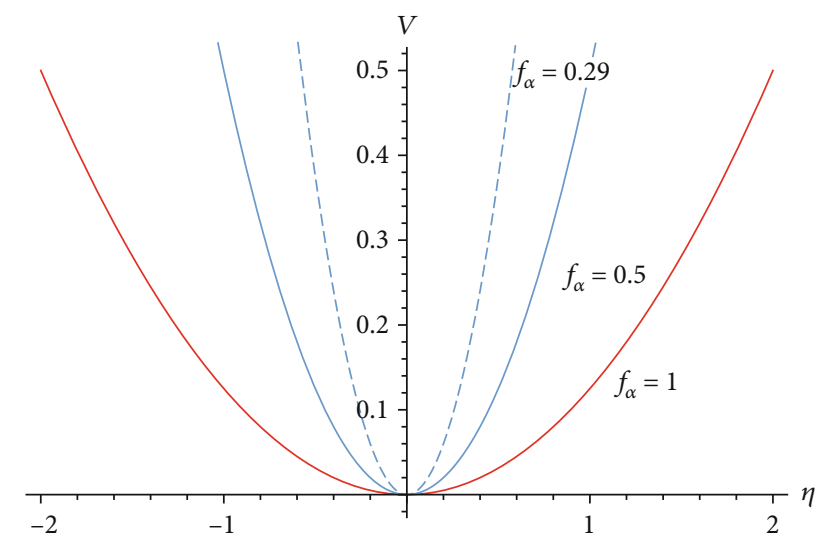

FIGURE 4: The glueball potential $V(\eta)$ against the glueball field $\eta$ for different values of $f_{\alpha}$. The potential has a nondegenerate and welldefined vacuum giving the glueballs a real mass $m_{\eta}$.

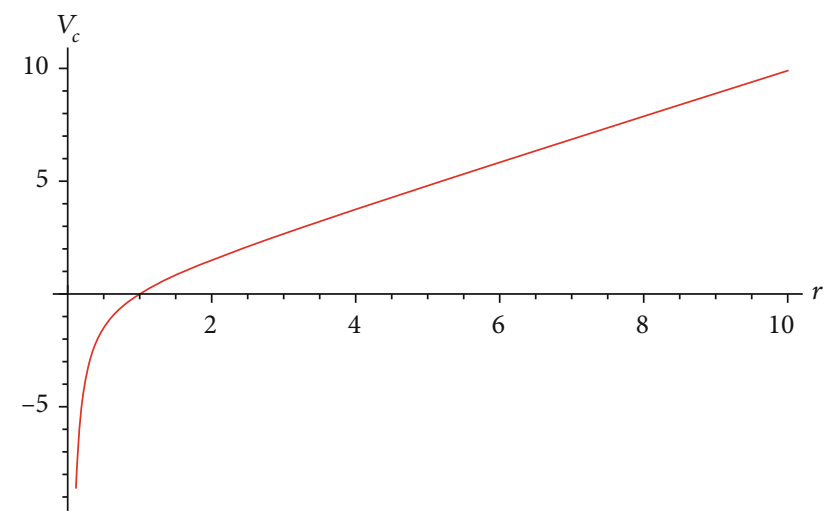

Figure 5: Confining Cornell-like potential. The perturbative and the nonperturbative nature of the potential is displayed. Below the Fermi scale, the gluons are asymptotically free, while above the Fermi scale, we only find confined color neutral particles.

here, $\overrightarrow{\widetilde{E}}$ and $\overrightarrow{\widetilde{B}}$ are the dual versions of the static electric and magnetic fields, while $\vec{j}_{m}$ and $\rho_{m}$ are the magnetic current and charge densities, respectively. The Lorentz force associ-

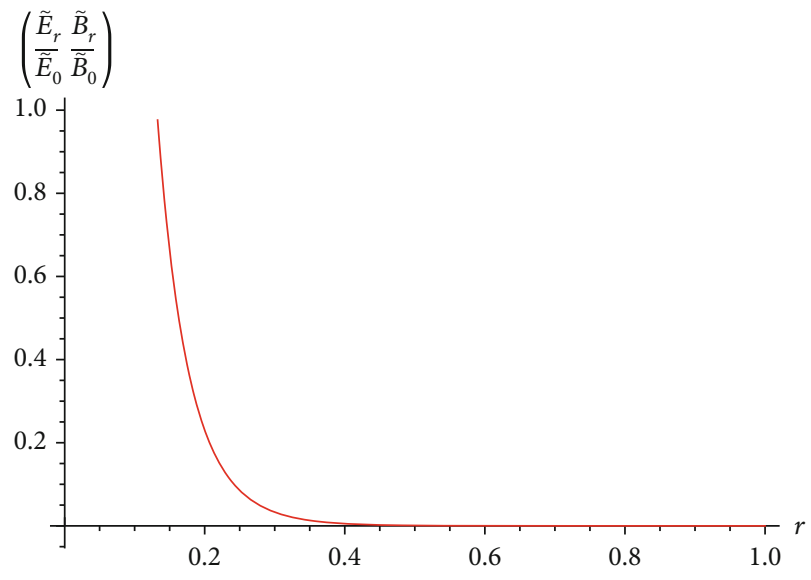

FIgURE 6: The screened electric (magnetic) field $\tilde{E}_{r}\left(\tilde{B}_{r}\right)$ against $r$. This graph holds for both electric and magnetic fields; thus, both fields are screened from the interior of the vacuum. It was sketched for $\lambda=65 \mathrm{~nm}$; below it, we have a mix or an intermediate state with higher electric or magnetic field strength. Above the estimated $\lambda$, we have a superconducting state because the vacuum is pure diamagnetic with no penetrating electric or magnetic fields.

ated with these fields can be expressed as

$$
\vec{F}=q(\overrightarrow{\widetilde{B}}-\vec{v} \times \overrightarrow{\tilde{E}})
$$

where $\vec{v}$ is the speed of the particle within the fields and $q$ is the monopole charge. The homogeneous equation $\nabla \cdot \overrightarrow{\vec{E}}=0$ represents the uniform electric field present at the confining phase. Combining the dual version of Ampere's law on the right side of Equation (65) with the dual London equation responsible for persistent current generated by the monopoles

$$
\nabla \times \vec{j}_{m}=\frac{1}{\lambda^{2}} \overrightarrow{\tilde{E}}
$$

we obtain the expression

$$
\nabla^{2} \overrightarrow{\widetilde{E}}=\frac{1}{\lambda^{2}} \overrightarrow{\widetilde{E}}
$$

Noting that $E=\nabla \times A$, we can identify $\lambda=\left(q^{2}\left|\phi_{0}\right|^{2}\right)^{-1 / 2}$ $=\left(q^{2} m_{\eta}^{2} / 2 \alpha^{4} \rho\right)^{-1 / 2}$ as the London penetration depth [112, 113]. Developing the Laplacian in Equation (68) in spherical coordinates yields

$$
\frac{d^{2} \tilde{E}_{r}}{d r^{2}}+\frac{2}{r} \frac{d \tilde{E}_{r}}{d r}-\frac{\tilde{E}_{r}}{\lambda^{2}}=0 ;
$$

this equation has a solution given as

$$
\tilde{E}_{r}=\frac{c_{1} e^{-r / \lambda}}{r}
$$




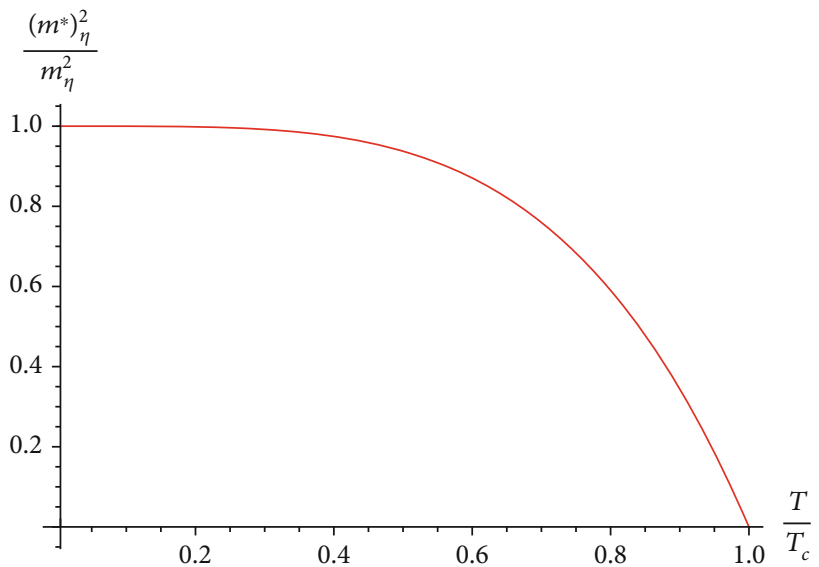

FIgURE 7: Thermally fluctuating glueball mass with temperature. The glueball mass decreases sharply with increasing $T$ until it vanishes at $T=T_{c}$, where the glueballs melt into gluons.

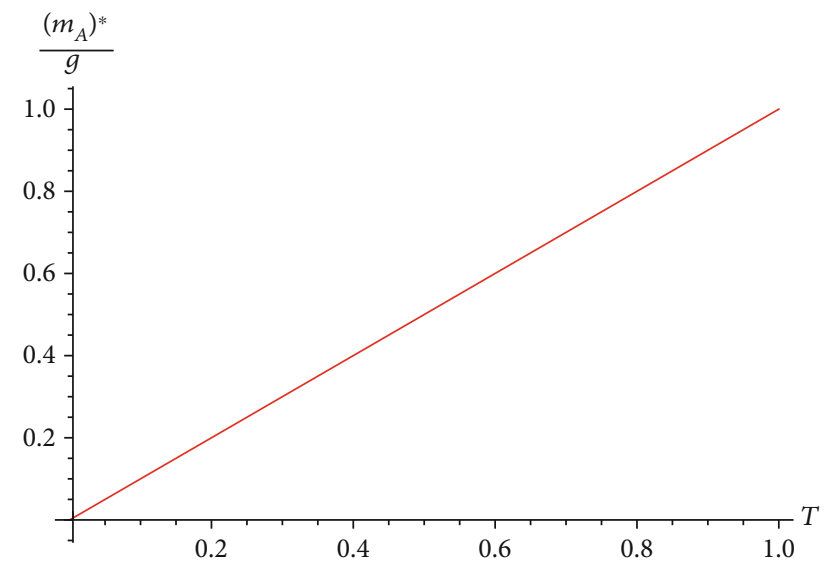

FIgURE 8: Variation of the gluon mass $m_{A}^{*}(T) / g$ with $T$. The gluon mass rather increases with temperature, because this phenomenon occurs at a temperature where the bound states of gluons or glueballs melt into gluons.

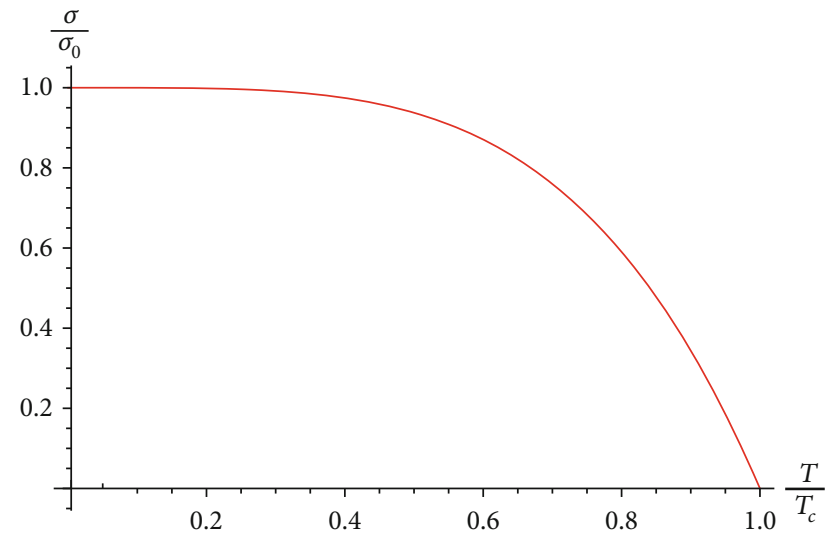

Figure 9: The string tension $\sigma(T) / \sigma_{0}$ against temperature $T / T_{c}$. The string tension decreases sharply with $T$ and breaks or vanishes at $T=T_{c}$ representing hadronization.

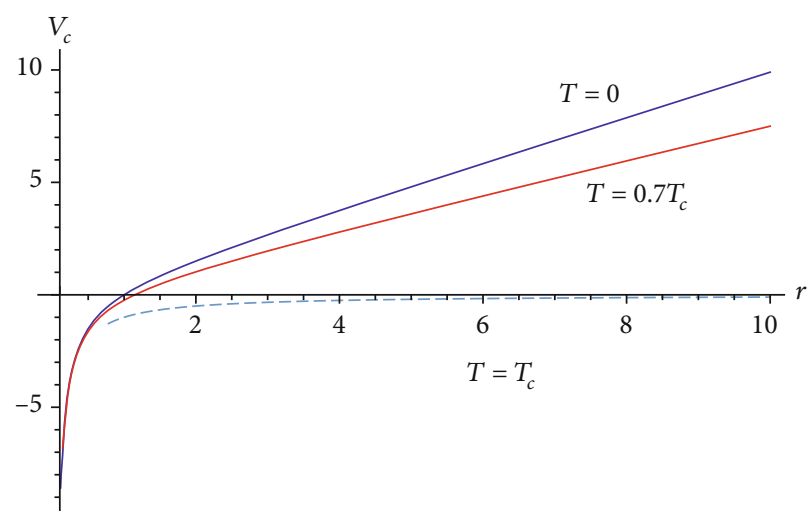

Figure 10: Temperature fluctuating Cornell-like potential. The gradient of the graph decreases with increasing $T$ and flattens at $T$ $=T_{c}$ indicating deconfinement and hadronization phase.

where $c_{1}$ is a constant. Using dimensional analysis, we can fix the constant as $c_{1}=\widetilde{E}_{0}$; therefore,

$$
\tilde{E}_{r}=\frac{\tilde{E}_{0} e^{-r / \lambda}}{r},
$$

where $\tilde{E}=|\tilde{E}|=\tilde{E}_{r}$. This means that the electric field is exponentially screened from the interior of the vacuum with penetration depth $\lambda$, a phenomenon known as the color Meissner effect. Also, using London's accelerating current relation,

$$
\overrightarrow{\widetilde{B}}=-\lambda^{2} \frac{\partial \vec{j}_{m}}{\partial t},
$$

and writing the continuity equation in the form of London equations,

$$
\frac{\partial \vec{j}_{m}}{\partial t}+\nabla \rho_{m}=0
$$

together with the equation at the left side of Equation (65), we obtain

$$
\nabla^{2} \overrightarrow{\widetilde{B}}=\frac{\overrightarrow{\tilde{B}}}{\lambda^{2}}
$$

This equation has a solution similar to Equation (69), i.e.,

$$
\tilde{B}_{r}=\frac{\tilde{B}_{0} e^{-r / \lambda}}{r} .
$$

Thus, the magnetic field is equally screened exponentially from the interior of the vacuum by a penetration depth $\lambda$. In sum, both time-varying electric and magnetic fields are screened with the same depth $\lambda$. 


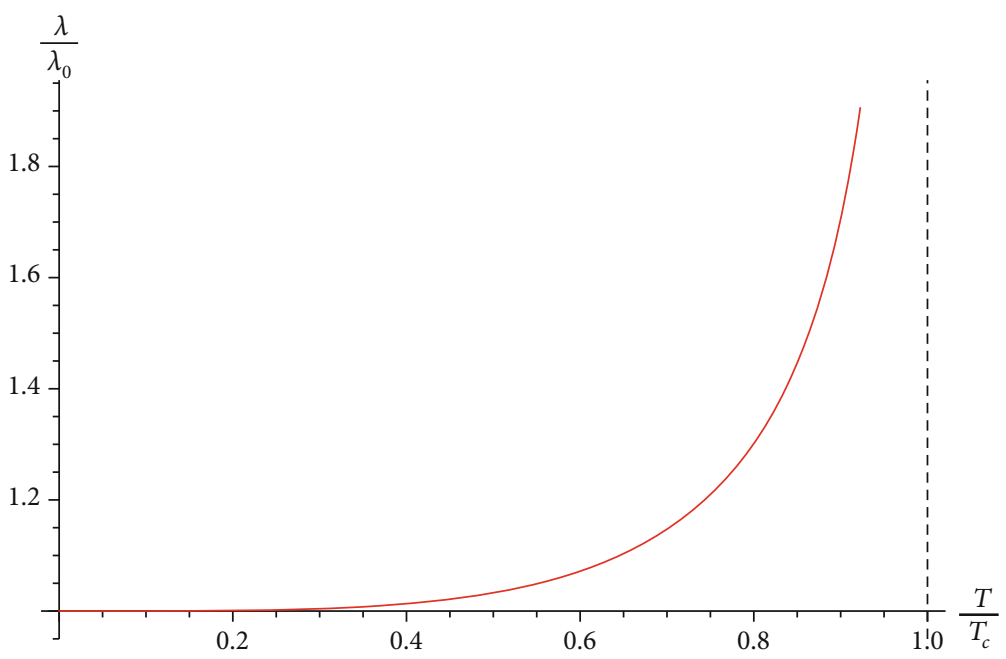

(a)

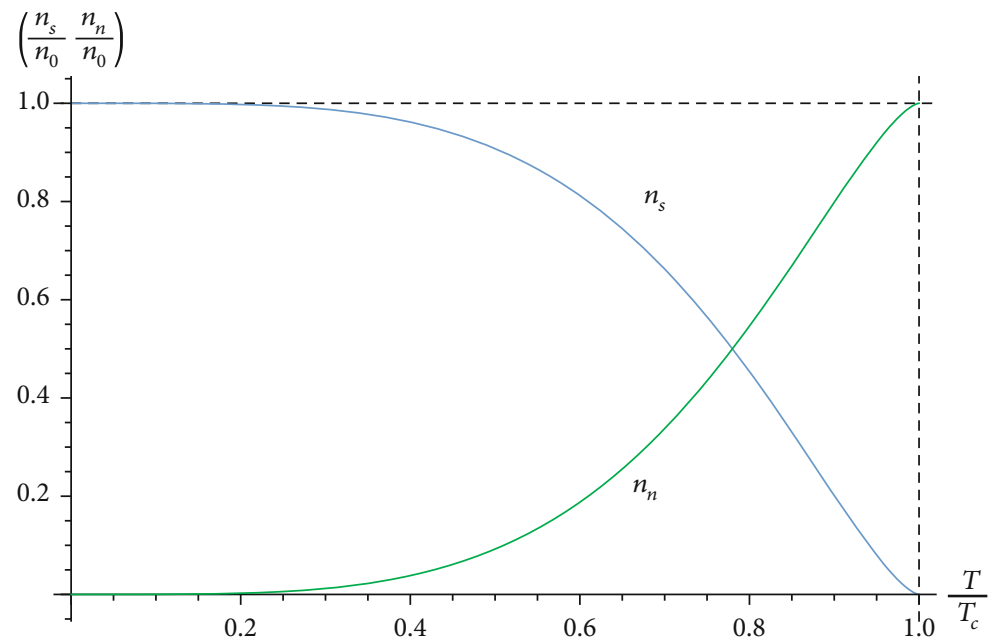

(b)

Figure 11: Penetration depth $\lambda$ (a), superconducting $n_{s}$, and normal $n_{n}$ monopole densities with temperature (b). The penetration depth remains constant at $\lambda=\lambda_{0}$ representing pure diamagnetic vacuum before it starts rising steadily with $T$ and becomes infinite at $T=T_{c}$, where the fields are expected to penetrate the vacuum. Thus, we have a superconducting state at low temperatures, and at relatively high temperatures, we have a mix or intermediate state. On the other hand, the superconducting monopole density $n_{s}$ decreases with increasing $T$ while the normal monopole density $n_{n}$ increases with an increasing $T$.

Now, comparing $\lambda$ with the results in Refs. $[112,114]$, where

$$
\lambda^{2}=\frac{m}{n_{s} e^{2}}
$$

we rearrange the expression obtained for $\lambda$ in the model framework,

$$
\lambda^{2}=\frac{2 \alpha^{4} \rho}{q^{2} m_{\eta}^{2}}=\frac{m_{\eta}^{2}}{2 q^{2} \rho a^{4}}
$$

to make it suitable for comparison. Juxtaposing these two equations leads to $q=e, m=m_{\eta}^{2} / a$ and $n_{s}=2 \rho a^{3}$, where $m, e$ , and $n_{s}$ are mass, charge, and electron number density of a superconducting material. Hence, the electron charge $e$ is equivalent to the monopole charge $q[115,116]$, and the electron number density and mass are related to the monopole density and mass, respectively. Combining Equation (67) with the electric field expression at the right side of Equation (65), we obtain a fluxoid quantization relation:

$$
\int \overrightarrow{\widetilde{E}} \cdot d \vec{S}-\lambda^{2} \oint \vec{j}_{m} \cdot d \vec{l}=n \Phi_{e}
$$

where $n \in \mathbb{Z}$ and $\Phi_{e}$ is the quantum of the electric flux [112]. The penetration depth could also be expressed as a function of temperature

$$
\lambda(T) \simeq\left(\frac{2 \alpha^{4} \rho}{q^{2} m_{\eta}^{* 2}(T)}\right)^{1 / 2} \simeq \lambda(0)\left[1-\frac{T^{4}}{T_{c}^{4}}\right]^{-1 / 2} .
$$


We retrieve the initial penetration depth below Equation (68), if we set $T=0$. Accordingly, the penetration depth increases with temperature until it becomes infinite at $T=$ $T_{c}$, where deconfinement and restoration of chiral symmetry coincide $[117,118]$. At this point, the field lines are expected to penetrate the vacuum causing it to lose its superconducting properties. This expression is precisely the same as the one obtained for metallic superconductors [114]. At $T>T_{c}$, the magnetic and electric field lines go through the vacuum. This phenomenon is referred to as the Meissner effect. The number density of the superconducting monopoles can also be expressed in terms of temperature as

$$
n_{s}(T)=2 \rho a^{3}\left[1-\frac{T^{4}}{T_{c}^{4}}\right]^{3 / 2}=n\left[1-\frac{T^{4}}{T_{c}^{4}}\right]^{3 / 2}
$$

The number density decreases sharply with temperature $T$ and vanishes at $T=T_{c}$. On the other hand, at $T<T_{c}$, similar to the two-fluid model where normal monopole fluid mixes with superconducting ones, the density of the monopoles can be calculated from the relation $n=n_{s}+n_{n}$, where $n_{n}$ is the normal monopole density [119]. Additionally, at $T$ $\longrightarrow 0, n_{s} \longrightarrow n$, and in the limit $T \longrightarrow T_{c}, n_{s} \longrightarrow 0$. Consequently,

$$
n_{n}=n\left\{1-\left[1-\frac{T^{4}}{T_{c}^{4}}\right]^{3 / 2}\right\}
$$

Thus, the color superconducting monopoles $n_{s}$ at $T<T_{c}$ conduct with dissipationless flow; on the other hand, the normal monopoles $n_{n}$ conduct with finite resistance at the same temperature range. Consequently, $n_{s}$ decreases sharply with increasing $T$ while $n_{n}$ increases steadily with increasing $T$.

\section{Analysis and Conclusion}

5.1. Analysis. At a high energy regime, there is high particle density which forms deconfined matter leading to hadronization $[120,121], \phi^{*}\left(p_{1}\right) \phi\left(p_{2}\right) \longrightarrow \gamma \longrightarrow \phi^{*}\left(p_{1}^{\prime}\right) \phi\left(p_{2}^{\prime}\right)[68,122-$ $125]$. In this regime, the process takes place at low temperatures but in high momentum and particle density. This leads to an asymptotic freedom behaviour among the particles because color confinement depends on how far or close the particles are together. Thus, higher density means the particles are closer together leading to deconfinement. Additionally, Equation (55) represents the gluon propagator which mediates the interaction of the glueballs in the IR regime of the model. Hadronization [126] comes into play in this regime when the momenta of the particles are reduced below a particular threshold set by the QCD scale, $\Lambda_{\mathrm{QCD}} \sim 250$ $\mathrm{MeV}$, or the separation distance between particle and antiparticle pairs is greater than $\sim 1 \mathrm{fm}$. For the sake of clarity, there are two forms of hadronization mentioned here, one arising from high energy QGP formation and the other from QCD string decay into hadrons in the low energy regime. The QGP hadronization is believed to have been formed immediately after the Big Bang when the QGP starts cooling down to
Hagedorn temperature, $T \sim 170 \mathrm{MeV}$, or higher baryon densities above $5 \rho_{0}$ where the free quarks and gluons start clamping together into hadrons. This is also observed at the initial stages of heavy ion collisions [121, 127, 128]. On the other hand, the string decay hadronization occurs due to nonperturbative effects. Under this picture, new hadrons are formed out of quark-antiquark pairs or through a gluon cascade [126].

Theoretically, Light-Front (LF) is one of the suitable theories for describing relativistic interactions due to its natural association with the light cone. Hence, under this theory, one can perform Fock expansion

$$
|\pi\rangle=|q \bar{q}\rangle+|q \bar{q} q \bar{q}\rangle+|q \bar{q} g\rangle+\cdots
$$

where the valence $|q \bar{q}\rangle$ and the nonvalence quark components are fully covered $[129,130]$. Hence, there is much information on the partonic structure of hadrons stored in the pions produced during hadronization that can be further exploited. These processes give an insight into the transition to the hypothetical QGP phase of matter where deconfinement and chiral symmetry restoration are expected. These studies gained more attention when ultrarelativistic heavy ion collision experiments in BNL Brookhaven and CERN Geneva were able to create matter under extreme conditions of temperature and densities necessary for phase transition [131]. Pion-pion annihilation is particularly necessary since pions are the second most abundant particles produced during the heavy ion collision from hot and dense hadron gas. Such annihilation also results in the production of photon and dilepton which form the basics for probing quarkgluon plasma phase and further hadronizations. These particles are known to leave the dense matter phase quickly without undergoing strong interactions [10-12]. However, the leptons are capable of annihilating into quarks and gluons (partons) in a process popularly referred to as gluon bremsstralhaung $[16,17]$. The free parameters of the model are $v$, $\xi,\left|\varepsilon_{v}\right|, \rho, \alpha, a$, and $\phi_{0}$, where $v$ is the degeneracy of the gluons and its acceptable value for massless gluons in SU(3) representation is $v=16$ while its value under $\mathrm{SU}(2)$ representation is $v=6$. It is known from QCD lattice simulation that $\sigma \sim 1$ $\mathrm{GeV} / \mathrm{fm}$; therefore, we can determine from Equation (28) that $m_{\eta}=1.73 \mathrm{GeV}[43-45,132,133]$ which is precisely the scalar glueball mass. For zero degeneracy, i.e., $v=0$, the critical temperature Equation (34) goes to infinity and all the glueballs get melted leaving pure gluons [75]. We determined the gluon mass to be $m_{A}=870 \mathrm{MeV}$ in the model framework. This value lies within the values obtained from lattice simulation as referred to in Section 3.3. Also, $\left|\varepsilon_{v}\right|$ is the magnitude of the QCD vacuum at the ground state. It has different values depending on the model under consideration. Its value estimated from the sum rule for gluon condensate is $0.006 \pm$ $0.012 \mathrm{GeV}^{4}$ [134], and the Bag model is also quoted as $(145)^{4} \mathrm{MeV}^{4}$. From the model $\left|\varepsilon_{v}\right|=\left\langle F^{\mu \nu} F_{\mu \nu}\right\rangle=\xi^{-2}=\rho a^{4}$, recalling that $\phi_{0}$ is related to $a$ and $\alpha$ as $\phi_{0}=a / \alpha, a$ has the dimension of energy and $\alpha$ is dimensionless. Again, when we combine Equations (28) and (37), we can express the 
string tension $\sigma$ as a function of temperature $T$ :

$$
\sigma(T)=\frac{m_{\eta}^{* 2}(T)}{3}=\frac{m_{\eta}^{2}(0)}{3}\left[1-\frac{T^{4}}{T_{c}^{4}}\right]
$$

Here, we retrieve Equation (28) at $T=0, \sigma\left(T=T_{c}\right)=0$, and at $T>T_{c}$, we move into the quark-gluon plasma (QGP) phase where the particles interact in a disorderly manner $[46,135,136]$. Thus, the potential in Equation (27) can be expressed in terms of temperature as

$$
V_{c}(r, T)=-\frac{1}{r}+\frac{m_{\eta}^{* 2}(T)}{3} r=-\frac{1}{r}+\frac{m_{\eta}^{2}(0)}{3}\left[1-\frac{T^{4}}{T_{c}^{4}}\right] r,
$$

where we recover Equation (27) at $T=0$. Thermal deconfinement and the restoration of chiral symmetry occur at $T=T_{c}$ $[117,118]$ due to the dissolution of the glueball mass, and we have the QGP phase at $T>T_{c}[46,135,136]$ where the glueball mass becomes unstable. As established in $\mathrm{SU}(2)$ group representation, it is known from the hadron spectrum that the $\mathrm{SU}(2)_{L} \times \mathrm{SU}(2)_{R}$ chiral symmetry is spontaneously broken due to the nonperturbative dynamics of the theory, leading to color confinement. Considering quark representation in $\mathrm{SU}(2)$, the color charges may be screened by the gauge degrees of freedom. Under this representation, we can possibly have quark-gluon color singlet thermionic states, quarkantiquark color singlets, or gluon-gluon color singlets. Generally, in this paper, we investigate glueballs which will fall under the gluon-gluon color singlet states.

5.2. Conclusion. The process of high energy annihilation of $\phi^{*} \phi$ to the production of $\phi^{*} \phi$ through the interaction $\phi^{*} \phi$ $\longrightarrow \gamma \longrightarrow \phi^{*} \phi$ during hadronization in the high particle density region is briefly addressed. Thus, we focus on the discussion to cover the low energy regime where $\tilde{U}(1)$ symmetry is spontaneously broken through the Abelian Higgs mechanism to give mass to the resulting glueballs. The scalar field in this case plays the role of the Higgs field, which undergoes modification into glueballs with mass $m_{\eta}$. This leads to color confinement of glueballs. We explored the behaviour of the scalar field potential $V(|\phi|)$ and the glueball potential $V(\eta)$ with the decay constant $f_{\alpha}$, and the results are presented in Figures 3 and 4. The model explains other confining (IR) properties such as gluon condensation, glueball mass, gluon mass, string tension, and dual superconductivity through monopole condensation. The results for color confinement, screened electric (magnetic) field due to the monopoles, and the gluon condensate are presented in Figures 1-5, respectively. The glueball fields acquire their mass through SSB, and their mass remains the most relevant parameter throughout the confinement properties. The magnitude of the glueball mass $m_{\eta}$ is precisely the same as the observed lightest scalar glueball mass $m_{\eta}=1.73 \mathrm{GeV}$. This mass is responsible for the string tension $\sigma$ that keeps the particles in a confined state and appears in the monopole condensate as well. It also appears in the gluon condensate $\left\langle 2 G(\eta) F^{\mu \nu} F_{\mu \nu}\right\rangle$, capable of hadronizing to form light pions in the low energy regime. In this paper, two forms of hadronization were discussed; the first one takes place at the high energy regime where the individual quarks and gluons recombine to form hadrons/pions. The second is at the low energy regime where the quark and antiquark pairs/valence gluons that form pions/glueballs get separated due to long separation distances, and the string tension that holds them together breaks leading to hadronization. We also investigated the effect of temperature on the effective scalar glueball mass $m_{\eta}^{* 2}(T)$, the gluon mass $m_{A}^{*}(T)$, string tension $\sigma(T)$, confining potential $V_{c}(r, T)$, gluon condensate $\left\langle 2 G(\eta) F^{\mu \nu} F_{\mu \nu}\right\rangle_{T}$, the penetration depth $\lambda(T)$, the superconducting monopole densities $n_{s}(T)$, and the normal monopole density $n_{n}(T)$, and their results are presented in Figures 611 , respectively. We calculated the QCD $\beta$-function and the strong "running" coupling $\alpha_{s}$ through renormalization group theory to enhance the discussions on gluon mass generation. Additionally, the glueball mass and the gluon masses were calculated, and the outcome was compared with lattice simulation results, analytical study, or phenomenological analyses to ascertain their reasonability. Finally, the model produces the correct behaviour of confining potential at $T=0$, where the potential has linear growth with $r$, Equation (27), consistent with the Cornell potential model. The potential keeps growing linearly for $T<T_{c}$ with thermal deconfinement phase at $T \geq T_{c}$. However, most of the results on QCD lattice calculations point to a temperature correction of order $-T^{2}$ to the string tension [137-139]; meanwhile, the model proposes correction in order of $-T^{4}$ in Equation (83). This can be regularized at $T \simeq T_{c}$ to obtain the correct order $-T^{2}$. Indeed, we did not find such regularization necessary because there are some proposed phenomenological models that suggest $-T^{4}$ correction [140] to the string tension as well. But we deem it necessary to provide some explanations based on the model. In the previous paper Ref. [40], we obtained a correction of order $-T^{2}$ as elaborated in Equation (63). We observed that in Ref. [40], the temperature correction to the string tension came from the scalar field $\phi$ as elaborated in the latter part of Section 3.3. On the other hand, the temperature correction in the gauge field leads to a correction in the order of $-T^{4}$ as discussed in Section 3.2 and elaborated in Section 5.1 in Equations (83) and (84).

\section{Data Availability}

The data used to support the findings of this study have been made already available in the literature.

\section{Conflicts of Interest}

The authors declare that there are no conflicts of interest regarding the publication of this article.

\section{Acknowledgments}

We would like to thank CNPq, CAPES, and CNPq/PRONEX/FAPESQ-PB (Grant no. 165/2018), for partial financial 
support. FAB also acknowledges support from CNPq (Grant no. $312104 / 2018-9)$.

\section{References}

[1] S. Marzani, G. Soyez, and M. Spannowsky, Lecture Notes in Physics, 2019.

[2] A. Ali and G. Kramer, "JETS and QCD: a historical review of the discovery of the quark and gluon jets and its impact on QCD," The European Physical Journal H, vol. 36, no. 2, pp. 245-326, 2011.

[3] R. Kogler, B. Nachman, A. Schmidt et al., "Jet substructure at the large hadron collider," Reviews of Modern Physics, vol. 91, no. 4, article 45003, 2019.

[4] W. Bartel, T. Canzler, D. Cords et al., "Observation of planar three-jet events in $\mathrm{e}^{+} \mathrm{e}^{-}$annihilation and evidence for gluon bremsstrahlung," Physics Letters B, vol. 91, no. 1, pp. 142147, 1980.

[5] C. Berger, H. Genzel, R. Grigull et al., "Evidence for gluon bremsstrahlung in $\mathrm{e}^{+} \mathrm{e}^{-}$annihilations at high energies," Physics Letters B, vol. 86, no. 3-4, pp. 418-425, 1979.

[6] D. P. Barber, U. Becker, H. Benda et al., "Discovery of threejet events and a test of quantum chromodynamics at PETRA," Physical Review Letters, vol. 43, no. 12, pp. 830833, 1979.

[7] R. Brandelik, W. Braunschweig, K. Gather et al., "Evidence for planar events in $\mathrm{e}^{+} \mathrm{e}^{-}$annihilation at high energies," Physics Letters B, vol. 86, no. 2, pp. 243-249, 1979.

[8] S. Abachi, B. Abbott, M. Abolins et al., "Observation of the top quark," Physical review Letters, vol. 74, no. 14, pp. 2632-2637, 1995.

[9] CDF Collaboration, "Observation of top quark production in Pbar-P collisions,” 1995, https://arxiv.org/abs/hep-ex/ 9503002.

[10] G. Chanfray, R. Rapp, and J. Wambach, "Medium modifications of the rho meson at CERN super proton synchrotron energies (200 GeV/nucleon)," Physical Review Letters, vol. 76, no. 3, pp. 368-371, 1996.

[11] G. Q. Li, C. M. Ko, and G. E. Brown, "Enhancement of lowmass dileptons in heavy ion collisions," Physical Review Letters, vol. 75, no. 22, pp. 4007-4010, 1995.

[12] H. J. Schulze and D. Blaschke, "Dilepton enhancement by thermal pion annihilation in the CERES experiment," Physics Letters B, vol. 386, no. 1-4, pp. 429-436, 1996.

[13] M. K. Volkov, E. A. Kuraev, D. Blaschke, G. Ropke, and S. Schmidt, "Excess low energy photon pairs from pion annihilation at the chiral phase transition," Physics Letters B, vol. 424, no. 3-4, pp. 235-243, 1998.

[14] G. Hanson, G. S. Abrams, A. M. Boyarski et al., "Evidence for jet structure in hadron production bye $\mathrm{e}^{-}$annihilation," Physical Review Letters, vol. 35, no. 24, pp. 1609-1612, 1975.

[15] R. F. Schwitters, A. M. Boyarski, M. Breidenbach et al., "Azimuthal asymmetry in inclusive hadron production bye $\mathrm{e}^{+-}$ $\mathrm{e}^{-}$annihilation," Physical Review Letters, vol. 35, no. 20, pp. 1320-1322, 1975.

[16] S. L. Wu, "Discovery of the first Yang-Mills gauge particle The gluon," International Journal of Modern Physics A, vol. 30, no. 34, article 1530066, 2015.

[17] B. H. Wiik, "First results from PETRA," in Proceedings of Neutrino 79, International Conference on Neutrinos, Weak Interactions and Cosmology, Volume 1, Bergen, 1979.
[18] S. L. Wu and H. Zobernig, "A three-jet candidate (run 447, event 13177)," TASSO Note No. 84, 1979.

[19] F. Englert and R. Brout, "Broken symmetry and the mass of gauge vector mesons," Physical Review Letters, vol. 13, no. 9, pp. 321-323, 1964.

[20] P. W. Higgs, "Broken symmetries, massless particles and gauge fields," Physical Review, vol. 12, no. 2, pp. 132-133, 1964.

[21] P. W. Higgs, "Broken symmetries and the masses of gauge bosons," Physical Review Letters, vol. 13, no. 16, pp. 508509, 1964.

[22] G. S. Guralnik, C. R. Hagen, and T. W. B. Kibble, "Global conservation laws and massless particles," Physical Review Letters, vol. 13, no. 20, pp. 585-587, 1964.

[23] P. W. Higgs, "Spontaneous symmetry breakdown without massless bosons," Physical Review, vol. 145, no. 4, pp. 11561163, 1966.

[24] T. W. B. Kibble, "Symmetry breaking in non-Abelian gauge theories," Physical Review Letters, vol. 155, p. 1554, 1967.

[25] G. Aad, T. Abajyan, B. Abbott et al., "Observation of a new particle in the search for the standard model Higgs boson with the ATLAS detector at the LHC," Physics Letters B, vol. 716, no. 1, pp. 1-29, 2012.

[26] S. Chatrchyan, V. Khachatryan, A. M. Sirunyan et al., "Observation of a new boson at a mass of $125 \mathrm{GeV}$ with the CMS experiment at the LHC," Physics Letters B, vol. 716, no. 1, pp. 30-61, 2012.

[27] V. Koch and C. Song, "Dilepton production in ultrarelativistic heavy-ion collisions," Physical Review C, vol. 54, no. 4, pp. 1903-1917, 1996.

[28] K. L. Haglin, "Excess electron pairs from heavy-ion collisions at CERN and a more complete picture of thermal production," Physical Review C, vol. 53, no. 6, pp. R2606-R2609, 1996.

[29] D. Anchishkin and R. Naryshkin, "Pion and quark annihilation mechanisms of dilepton production in relativistic heavy ion collisions," Modern Physics Letters A, vol. 20, no. 27, pp. 2047-2055, 2005.

[30] G.'t. Hooft, "Topology of the gauge condition and new confinement phases in non-Abelian gauge theories," Nuclear Physics B, vol. 190, no. 3, pp. 455-478, 1981.

[31] Z. F. Ezawa and A. Iwazaki, "Abelian dominance and quark confinement in Yang-Mills theories," Physical Review D, vol. 25, no. 10, pp. 2681-2689, 1982.

[32] H. Shiba and T. Suzuki, "Monopoles and string tension in SU(2) QCD," Physical Review D, vol. 333, no. 3-4, pp. 461466, 1994.

[33] T. Suzuki and I. Yotsuyanagi, "Possible evidence for Abelian dominance in quark confinement," Physical Review D, vol. 42, no. 12, pp. 4257-4260, 1990.

[34] J. D. Stack, W. W. Tucker, and R. J. Wensley, “The maximal Abelian gauge, monopoles, and vortices in $\mathrm{SU}(3)$ lattice gauge theory," Nuclear Physics B, vol. 639, no. 1-2, pp. 203222, 2002.

[35] V. G. Bornyakov, H. Ichie, Y. Mori et al., "Baryonic flux in quenched and two-flavor dynamical QCD after Abelian projection," Physical Review D, vol. 70, no. 5, article 054506, 2004.

[36] V. G. Bornyakov, H. Ichie, Y. Mori et al., "Dynamics of monopoles and flux tubes in two-flavor dynamical QCD," Physical Review D, vol. 70, article 074511, 2004. 
[37] G. S. Bali, V. Bornyakov, M. Muller-Preussker, and K. Schilling, "Dual superconductor scenario of confinement: a systematic study of Gribov copy effects," Physical Review D, vol. 54, no. 4, pp. 2863-2875, 1996.

[38] N. Sakumichi and H. Suganuma, "Perfect Abelian dominance of quark confinement in SU(3) QCD," Physical Review D, vol. 90 , no. 11, article 111501, 2014.

[39] A. Issifu and F. A. Brito, "Confinement of fermions in tachyon matter," Advances in High Energy Physics, vol. 2020, Article ID 1852841, 18 pages, 2020.

[40] A. Issifu and F. A. Brito, "The (de)confinement transition in tachyonic matter at finite temperature," Advances in High Energy Physics, vol. 2019, Article ID 9450367, 9 pages, 2019.

[41] A. Issifu and F. A. Brito, "Connement of fermions in tachyon matter at finite temperature," https://arxiv.org/abs/2012 .15102 .

[42] C. L. Basham, L. S. Brown, S. D. Ellis, and S. T. Love, "Energy correlations in electron-positron annihilation: testing quantum chromodynamics," Physical Review Letters, vol. 41, no. 23, pp. 1585-1588, 1978.

[43] C. J. Morningstar and M. Peardon, "Glueball spectrum from an anisotropic lattice study," Physical Review D, vol. 60, no. 3, article 034509, 1999.

[44] M. Loan, X. Q. Luo, and Z. H. Luo, "Monte Carlo study of glueball masses in the hamiltonian limit of SU(3) lattice gauge theory," International Journal of Modern Physics A, vol. 21, no. 13n14, pp. 2905-2936, 2006.

[45] Y. Chen, A. Alexandru, S. J. Dong et al., "Glueball spectrum and matrix elements on anisotropic lattices," Physical Review $D$, vol. 73, no. 1, article 014516, 2006.

[46] R. Pasechnik and M. Šumbera, "Phenomenological review on quark-gluon plasma: concepts vs. observations," Universe, vol. 3, no. 1, p. 7, 2017.

[47] T. Schäfer and E. Shuryak, "Instantons in QCD," Reviews of Modern Physics, vol. 70, no. 2, pp. 323-425, 1998.

[48] K. Melnikov and T. van Ritbergen, "The three-loop relation between the and the pole quark masses," Physics Letters $B$, vol. 482, no. 1-3, pp. 99-108, 2000.

[49] P. I. Fomin, V. P. Gusynin, V. A. Miransky, and Y. A. Sitenko, "Dynamical symmetry breaking and particle mass generation in gauge field theories," La Rivista Del Nuovo Cimento Series 3, vol. 6, no. 5, pp. 1-90, 1983.

[50] K. Higashijima, "Dynamical chiral-symmetry breaking," Physical Review D, vol. 29, no. 6, pp. 1228-1232, 1984.

[51] R. Acharya and P. Narayana-Swamy, "Dynamical realization of chiral symmetry in quantum chromodynamics," Physical Review D, vol. 26, no. 10, pp. 2797-2803, 1982.

[52] R. Acharya and P. Narayana-Swamy, "Dynamical breakdown of chiral symmetry in continuum QCD," Il Nuovo Cimento A, vol. 86, p. 157, 1985.

[53] R. Acharya and P. Narayana-Swamy, "The quark propagator in quantum chromodynamics: a study in Landau gauge," Zeitschrift für Physik C Particles and Fields, vol. 28, p. 463, 1985.

[54] M. Bando, T. Muta, and K. Yamawaki, Internationa1 Workshop on New Trends in Strong Coupling Gauge Theories, Nagoya, Japan, 1988, World Scientic, Singapore, 1989.

[55] J. Preskil, "Magnetic monopoles," Annual Review of Nuclear and Particle Science, vol. 34, no. 1, pp. 461-530, 1984.

[56] Y. Nambu, "Strings, monopoles, and gauge fields," Physical Review D, vol. 10, no. 12, pp. 4262-4268, 1974.
[57] G. 't Hooft, High Energy Physics, Compositori, Bologna, 1975.

[58] S. Mandelstam, "II. Vortices and quark confinement in nonAbelian gauge theories," Physics Reports, vol. 23, pp. 245-249, 1976.

[59] A. Iwazaki, "Monopoles and the Polyakov loop," Physical Review D, vol. 95, no. 9, article 094026, 2017.

[60] A. Di Giacomo and M. Hasegawa, "Instantons and monopoles," Physical Review D, vol. 91, no. 5, article 054512, 2015.

[61] A. Ramamurti and E. Shuryak, "Chiral symmetry breaking and monopoles in gauge theories," Physical Review D, vol. 100, article 016007, 2019.

[62] J. Liao and E. Shuryak, "Strongly coupled plasma with electric and magnetic charges," Physical Review C, vol. 75, no. 5, article 054907, 2007.

[63] J. Xu, J. Liao, and M. Gyulassy, "Consistency of perfect fluidity and jet quenching in semi-quark-gluon monopole plasmas," Chinese Physics Letters, vol. 32, no. 9, article 092501, 2015.

[64] A. Iwazaki, "Production of semi-quark-gluon-monopole plasma by glasma decay," Physical Review C, vol. 93, no. 5, article 054912, 2016.

[65] A. Dadi and C. Müller, "Phenomenological model of multiphoto-production of charged pion pairs on the proton," Physics Letters B, vol. 697, no. 2, pp. 142-146, 2011.

[66] F. Mandl and G. Shaw, Quantum Field Theory, The University of Manchester, Manchester, UK, 2nd edition, 2010.

[67] M. E. Peskin and D. V. Schroeder, An Introduction to Quantum Eld Theory, Westview Press, Chicago, USA, 1995.

[68] P. Langacker, The Standard Model and Beyond, CRC Pr, Boca Raton, USA, 2010.

[69] I. Low and Z. Yin, "The infrared structure of NambuGoldstone bosons," Journal of High Energy Physics, vol. 2018, no. 10, article 78, 2018.

[70] E. Klempt, C. Batty, and J.-M. Richard, "The antinucleonnucleon interaction at low energy: annihilation dynamics," Physics Reports, vol. 413, no. 4-5, pp. 197-317, 2005.

[71] N. A. Dondi, F. Sannino, and J. Smirnov, "Thermal history of composite dark matter," Physical Review D, vol. 101, no. 10, article 103010, 2020.

[72] W. Ochs, "The status of glueballs," Journal of Physics G: Nuclear and Particle Physics, vol. 40, no. 4, article 043001, 2013.

[73] F. E. Close and A. Kirk, "The mixing of the f0(1370), f0(1500) and $\mathrm{f} 0(1710)$ and the search for the scalar glueball," Physics Letters B, vol. 483, no. 4, pp. 345-352, 2000.

[74] S. J. Brodsky, A. S. Goldhaber, and J. Lee, "Hunting for glueballs in electron-positron annihilation," Physical Review Letters, vol. 91, no. 11, article 112001, 2003.

[75] G. W. Carter, O. Scavenius, I. N. Mishustin, and P. J. Ellis, "Effective model for hot gluodynamics," Physical Review C, vol. 61, no. 4, article 045206, 2000.

[76] M. A. Shifman, A. I. Vainshtein, and V. I. Zakharov, "QCD and resonance physics. Theoretical foundations," Nuclear Physics B, vol. 147, no. 5, pp. 385-447, 1979.

[77] D. Kharzeev, E. Levin, and K. Tuchin, "Classical gluodynamics in curved space-time and the soft pomeron," Physics Letters B, vol. 574, pp. 21-30, 2002.

[78] A. A. Migdal and M. A. Shifman, "Dilaton effective Lagrangian in gluodynamics," Physics Letters B, vol. 114, no. 6, pp. 445-449, 1982. 
[79] J. M. Cornwall and A. Soni, "Glueballs as bound states of massive gluons," Physics Letters B, vol. 120, no. 4-6, pp. 431-435, 1983.

[80] K.-I. Kondo, "Vacuum condensates, effective gluon mass and color confinement," in Color Confinement and Hadrons in Quantum Chromodynamics, Wako, Japan, 2004.

[81] D. B. Leinweber, J. I. Skullerud, A. Williams, and C. Parrinello, "Asymptotic scaling and infrared behavior of the gluon propagator," Physical Review D, vol. 60, no. 9, article 094507, 1999.

[82] K. Langfeld, H. Reinhardt, and J. Gattnar, "Gluon propagator and quark confinement," Nuclear Physics B, vol. 621, no. 1-2, pp. 131-156, 2002.

[83] C. Alexandrou, P. de Forcrand, and E. Follana, "Gluon propagator without lattice Gribov copies on a finer lattice," Physical Review D, vol. 65, no. 11, article 114508, 2002.

[84] J. H. Field, "Phenomenological analysis of gluon mass effects in inclusive radiative decays of the $J / \psi$ and $\Upsilon$," Physical Review D, vol. 66, no. 1, article 013013, 2002.

[85] M. Consoli and J. H. Field, "Effective gluon mass and the determination of $\alpha_{s}$ from $J / \psi$ and $\Upsilon$ branching ratios," Physical Review D, vol. 49, no. 3, pp. 1293-1301, 1994.

[86] D. B. Leinweber, J. I. Skullerud, A. G. Williams, and C. Parrinello, "Asymptotic scaling and infrared behavior of the gluon propagator," Physical Review D, vol. 60, no. 9, article 094507, 1999.

[87] I. I. Kogan and A. Kovner, "Variational approach to the QCD wave functional: dynamical mass generation and confinement," Physical Review D, vol. 52, no. 6, pp. 3719-3734, 1995.

[88] K. Kajantie, M. Laine, J. Peisa, A. Rajantie, K. Rummukainen, and M. Shaposhnikov, "Nonperturbative Debye mass in finite temperature QCD," Physical Review Letters, vol. 79, no. 17, pp. 3130-3133, 1997.

[89] S. Nadkarni, "Non-Abelian Debye screening: the coloraveraged potential," Physical Review D, vol. 33, no. 12, pp. 3738-3746, 1986.

[90] E. Manousakis and J. Polonyi, "Nonperturbative length scale in high-temperature QCD," Physical Review Letters, vol. 58, no. 9, pp. 847-850, 1987.

[91] A. Deur, S. J. Brodsky, and G. F. de Teramond, "The QCD running coupling," Progress in Particle and Nuclear Physics, vol. 90, pp. 1-74, 2016.

[92] J. Lan, C. Mondal, S. Jia, X. Zhao, J. P. Vary, and BLFQ Collaboration, "Pion and kaon parton distribution functions from basis light front quantization and QCD evolution," Physical Review D, vol. 101, no. 3, article 034024, 2020.

[93] Y. Liu and I. Zahed, "Pion condensation by rotation in a magnetic field," Physical Review Letters, vol. 120, no. 3, article 032001, 2018.

[94] P. Adhikari, J. O. Andersen, and P. Kneschke, "Pion condensation and phase diagram in the Polyakov-loop quark-meson model," Physical Review D, vol. 98, no. 7, article 074016, 2018.

[95] L. He, M. Jin, and P. Zhuang, "Pion superfluidity and meson properties at finite isospin density," Physical Review D, vol. 71, no. 11, article 116001, 2005.

[96] M. Alford, K. Rajagopal, and F. Wilczek, "QCD at finite baryon density: nucleon droplets and color superconductivity," Physics Letters B, vol. 422, no. 1-4, pp. 247-256, 1998.

[97] F. A. Brito and E. E. M. Lima, "Exploring the thermodynamics of non-commutative scalar elds," International Journal of Modern Physics A, vol. 31, no. 11, article 1650057, 2016.
[98] A. Barducci, G. Pettini, L. Ravagli, and R. Casalbuoni, "Ladder-QCD at finite isospin chemical potential," Physical Review Letters, vol. 564, no. 3-4, pp. 217-224, 2003.

[99] Y. Nishida, "Phase structures of strong coupling lattice QCD with finite baryon and isospin density," Physical Review D, vol. 69, no. 9, article 094501, 2004.

[100] S. Hands and D. N. Walters, "Numerical portrait of a relativistic BCS gapped superfluid," Physical Review D, vol. 69, no. 7, article 076011, 2004.

[101] L. He and P. Zhuang, "Phase structure of Nambu-JonaLasinio model at finite isospin density," Physics Letters B, vol. 615, no. 1-2, pp. 93-101, 2005.

[102] D. Toublan and J. B. Kogut, "Isospin chemical potential and the QCD phase diagram at nonzero temperature and baryon chemical potential," Physics Letters B, vol. 564, no. 3-4, pp. 212-216, 2003.

[103] A. Barducci, R. Casalbuoni, G. Pettini, and L. Ravagli, "Calculation of the QCD phase diagram at finite temperature, and baryon and isospin chemical potentials," Physical Review D, vol. 69, no. 9, article 096004, 2004.

[104] M. Sadzikowski, "Coexistence of pion condensation and color superconductivity in two flavor quark matter," Physics Letters B, vol. 553, no. 1-2, pp. 45-50, 2003.

[105] R. Rapp, T. Schäfer, E. Shuryak, and M. Velkovsky, "Diquark Bose condensates in high density matter and instantons," Physical Review Letters, vol. 81, no. 1, pp. 53-56, 1998.

[106] M. G. Alford, A. Schmitt, K. Rajagopal, and T. Schäfer, "Color superconductivity in dense quark matter," Reviews of Modern Physics, vol. 80, no. 4, pp. 1455-1515, 2008.

[107] J. Bardeen, L. Cooper, and J. R. Schrieffer, "Microscopic theory of superconductivity," Physical Review, vol. 106, no. 1, pp. 162-164, 1957.

[108] J. M. Carmona, M. D’Elia, L. del Debbio, A. di Giacomo, B. Lucini, and G. Paffuti, "Color confinement and dual superconductivity in full QCD,” Physical Review D, vol. 66, no. 1, article 011503, 2002.

[109] A. Ramamurti and E. Shuryak, "Chiral symmetry breaking and monopoles in gauge theories," Physical Review D, vol. 100, no. 1, article 016007, 2019.

[110] A. M. Polyakov, "Particle Spectrum in the Quantum Field Theory," JETP Letters, vol. 20, pp. 194-195, 1974.

[111] G. 't Hooft, "Magnetic monopoles in unified gauge theories," Nuclear Physics B, vol. 79, no. 2, pp. 276-284, 1974.

[112] V. Singh, D. A. Browne, and R. W. Haymaker, "Structure of Abrikosov vortices in SU(2) lattice gauge theory," Physics Letters B, vol. 306, no. 1-2, pp. 115-119, 1993.

[113] D. Bazeia, F. A. Brito, W. Freire, and R. F. Ribeiro, "Confinement from new global defect structures," The European Physical Journal C, vol. 40, no. 4, pp. 531-537, 2005.

[114] M. Tinkham, Introduction to Superconductivity, Dover Publications, Mineola, New York, 2004.

[115] P. A. M. Dirac, "Quantised singularities in the electromagnetic field," Proceedings of the Royal Society of London. Series A, Containing Papers of a Mathematical and Physical Character, vol. 133, pp. 60-72, 1931.

[116] J. Schwinger, "Magnetic charge and quantum field theory," Physical Review, vol. 144, no. 4, pp. 1087-1093, 1966.

[117] R. Gupta, G. Guralnik, G. W. Kilcup, A. Patel, and S. R. Sharpe, "Clear evidence for a first-order chiral transition in QCD,” Physical Review Letters, vol. 57, no. 21, pp. 26212624, 1986. 
[118] S. Gottlieb, W. Liu, D. Toussaint, R. L. Renken, and R. L. Sugar, "Chiral-symmetry breaking in lattice QCD with two and four fermion flavors," Physical Review D, vol. 35, no. 12, pp. 3972-3980, 1987.

[119] C. P. Poole, R. Prozorov, H. A. Farach, and R. J. Creswick, "Phenomenon of superconductivity," Superconductivity, vol. 33 , no. $85,2014$.

[120] J. Letessier and J. Rafelski, "Hadrons and quark-gluon plasma," Cambridge University Press, 2002.

[121] P. Senger, "Particle production in heavy-ion collisions," Progress in Particle and Nuclear Physics, vol. 53, no. 1, pp. 1-23, 2004.

[122] O. Linnyk, W. Cassing, and E. L. Bratkovskaya, "Centrality dependence of the direct photon yield and elliptic flow in heavy-ion collisions atsNN=200GeV," Physical Review C, vol. 89, no. 3, article 034908, 2014.

[123] W. Liu and R. Rapp, "Low-energy thermal photons from meson-meson bremsstrahlung," Nuclear Physics A, vol. 796, no. 1-4, pp. 101-121, 2007.

[124] E. L. Bratkovskaya, S. M. Kiselev, and G. B. Sharkov, "Direct photon production from hadronic sources in high-energy heavy-ion collisions," Physical Review C, vol. 78, no. 3, article 034905, 2008.

[125] V. Srinivasan, J. A. Helland, A. J. Lennox et al., " $\pi-\pi+\rightarrow \pi-\pi+$ interactions below $0.7 \quad \mathrm{GeV}$ from $\pi-\mathrm{p} \rightarrow \pi-\pi+$ ndata at $5 \mathrm{GeV} / \mathrm{c}$," Physical Review $D$, vol. 12, no. 3, pp. 681-692, 1975.

[126] B. Webber, "Hadronization," arXiv preprint hep-ph/9411384, 1994.

[127] P. Braun-Munzinger, K. Redlich, and J. Stachel, "Particle production in heavy ion collisions," in Quark-Gluon Plasma 3pp. 491-599, World Scientific Publishing Co Pte Ltd.

[128] M. Wagner, A. B. Larionov, and U. Mosel, "Kaon and pion production in relativistic heavy-ion collisions," Physical Review C, vol. 71, no. 3, article 034910, 2005.

[129] S. J. Brodsky, H.-C. Pauli, and S. S. Pinsky, "Quantum chromodynamics and other field theories on the light cone," Physics Reports, vol. 301, no. 4-6, pp. 299-486, 1998.

[130] G. Salmè, E. Pace, and G. Romanelli, "Exploring the pion phenomenology within a fully covariant constituent quark model," Few-Body Systems, vol. 54, no. 5-6, pp. 769-777, 2013.

[131] P. Braun-Munzinger, H. J. Specht, R. Stock, and H. Stöcker, "PrefacePreface," Nuclear Physics A, vol. 610, pp. xi-xii, 1996.

[132] W. Lee and D. Weingarten, "Scalar quarkonium masses and mixing with the lightest scalar glueball," Physical Review D, vol. 61, article 014015, 2000.

[133] G. S. Bali, K. Schilling, A. Hulsebos, A. C. Irving, C. Michael, and P. W. Stephenson, "A comprehensive lattice study of SU(3) glueballs," Physical Review B, vol. 309, no. 3-4, pp. 378-384, 1993.

[134] B. L. Ioffe, "Condensates in quantum chromodynamics," Physics of Atomic Nuclei, vol. 66, no. 1, pp. 30-43, 2003.

[135] K. Yagi, T. Hatsuda, and Y. Miake, "Quark-gluon plasma: from big bang to little bang," Cambridge monographs on particle physics, nuclear physics and cosmology, vol. 23, pp. 1-446, 2005.

[136] K. Yagi, T. Hatsuda, and Y. Miake, Cambridge Monographs on Particle Physics, Nuclear Physics and Cosmology, Cambridge University Press, Cambridge, UK, 2005.
[137] O. Kaczmarek, F. Karsch, E. Laermann, and M. Lutgemeier, "Heavy quark potentials in quenched QCD at high temperature," Physical Review D, vol. 62, no. 3, article 034021, 2000.

[138] R. D. Pisarski and O. Alvarez, "Strings at finite temperature and deconfinement," Physical Review D, vol. 26, no. 12, pp. 3735-3737, 1982.

[139] P. De Forcrand, G. Schierholz, H. Schneider, and M. Teper, "The string and its tension in $\mathrm{Su}(3)$ lattice gauge theory: towards definitive results," Physics Letters B, vol. 160, no. 13, pp. 137-143, 1985.

[140] H. Boschi-Filho, N. R. F. Braga, and C. N. Ferreira, "Heavy quark potential at finite temperature from gauge-string duality," Physics Letters B, vol. 74, no. 8, article 086001, 2006. 